\title{
Decompositions, Partitions, and Coverings with Convex Polygons and Pseudo-Triangles
}

O. Aichholzer ${ }^{1, \star}$, C. Huemer ${ }^{2, \star \star}$, S. Kappes ${ }^{3, \star \star \star}$, B. Speckmann ${ }^{4}$, and Cs. D. Tóth ${ }^{5}$

1 Institute for Software Technology, Graz University of Technology, Austria. e-mail: oaich@ist.tugraz.at

2 Departament de Matemática Aplicada II, Univ. Poli. de Catalunya, Spain. e-mail: clemens.huemer@upc.edu

3 Department of Mathematics, TU Berlin, Germany. e-mail:kappes@math.TU-Berlin.de

4 Department of Mathematics and Computer Science, TU Eindhoven, Netherland. e-mail: speckman@win.tue.nl

5 Department of Mathematics, MIT, Cambridege, MA 02144, USA. e-mail: toth@math.mit.edu

\begin{abstract}
We propose a novel subdivision of the plane that consists of both convex polygons and pseudo-triangles. This pseudo-convex decomposition is significantly sparser than either convex decompositions or pseudo-triangulations for planar point sets and simple polygons. We also introduce pseudo-convex partitions and coverings. We establish some basic properties and give combinatorial bounds on their complexity. Our upper bounds depend on new Ramsey-type results concerning disjoint empty convex $k$-gons in point sets.
\end{abstract}

Key words. Partitions, decompositions, planar point sets, Ramsey-type results.

\section{Introduction}

Geometric algorithms and data structures frequently use subdivisions of the input space into compact and easy to handle polygonal cells. Triangulations are among the most widely used of these tessellations. Since the running time of algorithms is often correlated with the size of the subdivision, many efficient algorithms tile the plane with generalizations of triangles such as convex polygons or pseudo-triangles which provide a sparser tessellation but retain many of the desirable properties of a triangulation. Both convex subdivisions and pseudo-triangulations have applications in areas like motion planning [7, 32], collision detection [1, 23], ray shooting [6, 15], or visibility [27-29]. A pseudo-triangle is the "most reflex" polygon possible - it

* Research partially supported by the FWF (Austrian Fonds zur Förderung der Wissenschaftlichen Forschung) under grant S09205-N12, FSP Industrial Geometry.

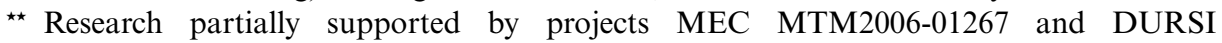
2005SGR00692.

$\star \star \star$ Research supported by the Deutsche Forschungsgemeinschaft within the European graduate program "Combinatorics, Geometry, and Computation" (No. GRK 588/2). 
has exactly three convex vertices with internal angles less than $\pi$. Whether a chain of points is considered convex or reflex depends only on the point of view. So pseudotriangles can be considered as natural counterparts of convex polygons.

In this paper we propose a combination of convex and pseudo-triangular subdivisions: Pseudo-convex decompositions. A pseudo-convex decomposition is a tiling of the plane with convex polygons and pseudo-triangles. We also introduce the related concepts of pseudo-convex partitions and coverings whose convex counterparts have been extensively studied as well. We establish some basic combinatorial properties and give quantitative bounds on the complexity of pseudo-convex decompositions, partitions, and coverings for point sets and simple polygons. Pseudo-convex decompositions are significantly sparser than convex decompositions or pseudo-triangulations.

All our bounds are combinatorial, in fact we do not know what the complexity of finding a minimum decomposition for a given input point set is. Our upper bounds depend on optimal solutions for small point configurations. Any improvement on a finite point set would lead to better bounds. We achieve optimal bounds for small configurations by proving two geometric Ramsey-type results concerning disjoint empty convex $k$-gons in point sets. These results extend previous work by Erdős, Hosono, and Urabe, but to the best of our knowledge our results are the first Ramsey-type answers to such questions. Small configurations of points are notoriously hard to deal with. An asymptotic lower bound for the number of order types of a set of $n$ points in the plane is $n^{\Theta(n \log n)}$ [14]. We confirmed our conjectures regarding sets of 8 and 11 points with the help of the order type data base developed at TU Graz [2,3]. We give analytical proofs for some of our results, while others are purely based on the data base.

Organization. The next paragraphs give precise definitions for convex and pseudoconvex decompositions, partitions, and coverings and Section 2 collects some of their basic combinatorial properties. In the next subsection we state our results and compare our bounds to previous work. Pseudo-convex decompositions and partitions are significantly sparser than their convex counterparts while pseudoconvex and convex coverings have asymptotically the same complexity. We devote Section 3 to pseudo-convex decompositions and Section 4 to pseudo-convex partitions of point sets. Subsection 3.1 formally states our two Ramsey-type theorems. Section 5 collects a number of observations concerning pseudo-convex coverings for small point sets. Finally, Section 6 discusses pseudo-convex decompositions for the interior of simple polygons. We conclude with some open problems.

Definitions. Let $S$ be a set of $n$ points in general position in the plane. A pseudotriangle is a planar polygon that has exactly three convex vertices with internal angles less than $\pi$, all other vertices are concave. A pseudo-triangulation of $S$ is a subdivision of the convex hull of $S$ into pseudo-triangles whose vertex set is exactly $S$. A vertex is called pointed if it has an adjacent angle greater than $\pi$. A planar straight line graph is pointed if every vertex is pointed.

The convex decomposition number of $S, \kappa_{d}(S)$, is the minimum number of faces in a subdivision of the convex hull of $S$ into convex polygons whose joint vertex set is exactly $S$. A pseudo-convex decomposition of $S$ is a subdivision of the convex 


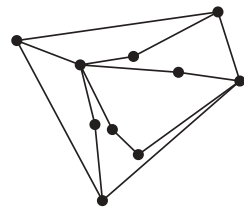

(a)

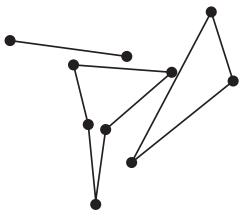

(b)

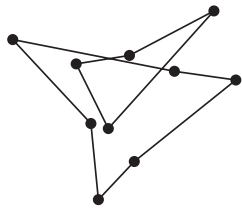

(c)

Fig. 1. A pseudo-convex decomposition (a), a pseudo-convex partition (b), and a pseudoconvex covering (c)

hull of $S$ into a family of convex polygons and pseudo-triangles whose joint vertex set is $S$. For instance every triangulation or pseudo-triangulation of $S$ is a pseudoconvex decomposition. The pseudo-convex decomposition number of $S, \psi_{d}(S)$, is the minimum number of faces in a pseudo-convex decomposition of $S$.

The convex partition number of $S, \kappa_{p}(S)$, is the minimum number of pairwise disjoint closed convex polygonal domains whose joint vertex set is $S$ (that is, the vertex sets of these convex polygons partition the point set $S$ ). Similarly, the pseudo-convex partition number of $S, \psi_{p}(S)$, is the minimum number of pairwise disjoint, closed, convex or pseudo-triangular polygonal domains whose joint vertex set is $S$. Note that each of the disjoint polygonal domains is empty (of points): neither a convex nor a pseudo-convex partition contains nested polygons.

The convex cover number of $S, \kappa_{c}(S)$, is the minimum number of convex polygons whose joint vertex set is $S$. Similarly, the pseudo-convex cover number of $S, \psi_{c}(S)$, is the minimum number of convex and pseudo-triangular polygons whose joint vertex set is $S$.

We are interested in combinatorial bounds on the maximum pseudo-convex decomposition (resp., pseudo-convex partition, pseudo-convex cover) number over all point sets of a given size $n \in \mathbb{N}$. We let

$$
\psi_{i}(n):=\max _{|S|=n} \psi_{i}(S), \quad \text { for } i=d, p, c .
$$

Similarly, for the convex decompositions (resp., partitions and coverings), we let $\kappa_{i}(n):=\max _{|S|=n} \kappa_{i}(S)$, for $i=d, p, c$.

\subsection{Previous Work and Results.}

Decomposition. The convex decomposition number $\kappa_{d}(n)$ is bounded by

$$
\frac{12}{11} n-2<\kappa_{d}(n) \leq \frac{10 n-18}{7} .
$$

The lower bound was given very recently by García-López and Nicolás [11] and the upper bound was established by Neumann-Lara et al. [26]. Fevens, Meijer, and Rappaport [10] and Spillner [31] designed algorithms for computing a minimum convex decomposition for input point sets. Every minimum pseudo-triangulation of $n$ points has exactly $n-2$ pseudo-triangles [32]. We show that the pseudo-convex decomposition number is bounded by 


$$
3\left\lfloor\frac{n}{5}\right\rfloor-1 \leq \psi_{d}(n) \leq \frac{7}{10} n .
$$

Furthermore, we also prove that $\psi_{d}(n)$ is monotonically increasing with $n$.

Partition. The convex partition number $\kappa_{p}(n)$ is bounded by

$$
\frac{n-1}{4} \leq \kappa_{p}(n) \leq\left\lceil\frac{5 n}{18}\right\rceil .
$$

The lower bound was given by Urabe [33] and the upper bound was established by Hosono and Urabe [18]. Arkin et al. [4] study questions related to convex partitions and coverings by examining the reflexivity of point sets. We show that the pseudo-convex partition number $\psi_{p}(n)$ is bounded by

$$
\frac{3}{4}\left\lfloor\frac{n}{4}\right\rfloor \leq \psi_{p}(n) \leq\left\lceil\frac{n}{4}\right\rceil .
$$

Covering. The study of convex cover numbers is rooted in the classical work of Erdös and Szekeres $[8,9]$ who showed that any set of $n$ points contains a convex subset of size $\Omega(\log n)$. More recent results include the work by Urabe [33] who proved that the convex cover number $\kappa_{c}(n)$ is bounded by

$$
\frac{n}{\log _{2} n+2}<\kappa_{c}(n)<\frac{2 n}{\log _{2} n-\log _{2} e} .
$$

There is an easy connection between the pseudo-convex cover number and the convex cover number, namely $\psi_{c}(n) \leq \kappa_{c}(n) \leq 3 \psi_{c}(n)$ (all points which can be covered by a pseudo-triangle can be covered by at most three convex sets). Thus both numbers have the same asymptotic behavior, which implies

$$
\psi_{c}(n)=\Theta\left(\frac{n}{\log n}\right) .
$$

Table 1 shows the exact values of $\psi_{c}(n), \psi_{p}(n)$, and $\psi_{d}(n)$ for small sets of points.

Geometric Ramsey-type Results. The upper bound construction for $\psi_{d}(n)$ relies on minimal pseudo-convex decomposition numbers for few points. These are, in turn, related to a combinatorial geometry problem on empty convex polygons that goes back to Erdös: For $k \geq 3$ find the smallest integer $E(k)$ such that any set $S$ of $E(k)$ points contains the vertex set of a convex $k$-gon whose interior does not contain any point of $S$. Klein [8] showed that every set of 5 points contains

Table 1. Bounds on the pseudo-convex cover number $\psi_{c}(n)$, partition number $\psi_{p}(n)$, and decomposition number $\psi_{d}(n)$ for small point sets

\begin{tabular}{llllllllllllll}
\hline$n$ & 3 & 4 & 5 & 6 & 7 & 8 & 9 & 10 & 11 & 12 & 13 & 14 & 15 \\
\hline$\psi_{d}(n)$ & 1 & 2 & 2 & 3 & 4 & 4 & 5 & 6 & 6 & 7 & 8 & $8 . .9$ & $8 . .9$ \\
$\psi_{p}(n)$ & 1 & 1 & 2 & 2 & 2 & 2 & 3 & 3 & 3 & 3 & $3 . .4$ & 4 & 4 \\
$\psi_{c}(n)$ & 1 & 1 & 2 & 2 & 2 & 2 & 2 & 3 & 3 & 3 & 3 & 3 & 3 \\
\hline
\end{tabular}


an empty convex quadrilateral, that is $E(4)=5$. Harborth [17] proved that every set of 10 points contains an empty convex pentagon, that is $E(5)=10$. In the last decade, Urabe [33] proved that every set of 7 points can be partitioned into a triangle and a disjoint convex quadrilateral. Hosono and Urabe [18] showed that every set of 9 points contains two disjoint empty convex quadrilaterals. Very recently Gerken [13, 34 ] showed that any set that contains a convex 9-gon also contains an empty convex hexagon. Each of these results corresponds to a bound on the pseudo-convex decomposition number $\psi_{d}(n)$. The best upper bound we achieved depends on new results for empty convex polygons.

A typical Ramsey type problem asks for the minimum size of a system that contains at least one of two (or more) subconfigurations. The classical Ramsey number $R(n, m)$ is the smallest integer such that every red-blue complete graph on $R(n, m)$ vertices contains a red $K_{n}$ or a blue $K_{m}$. The first geometric Ramsey-type problems focused on geometric graphs [19, 20] and intersection graphs [24].

We prove the following two results: (1) Every set of 8 points in general position contains either an empty convex pentagon or two disjoint empty convex quadrilaterals. (2) Every set of 11 points in general position contains either an empty convex hexagon or an empty convex pentagon and a disjoint empty convex quadrilateral.

Simple Polygons. An initial step of many algorithms on simple polygons is a decomposition into simpler components [21]. Keil and Snoeyink [22] devised an algorithm for computing the minimum convex decomposition of the interior of a given simple polygon. Chazelle and Dobkin [5] studied a variant of this optimization problem allowing Steiner points, Lien and Amato [25] constructed approximately convex decompositions. Motivated by our early results, obtained during our investigations for this paper, Gerdijkov and Wolff [12] extended the work by Keil and Snoeyink to compute the minimum pseudo-convex decomposition of a simple polygon.

The minimum convex decomposition of a pseudo-triangle with $n$ vertices may require $n-2$ triangles, and the minimum pseudo-triangulation of any convex $n$-gon is a triangulation with $n-2$ faces. (In these extremal examples, Steiner points do not lead to a smaller convex decomposition or pseudo-triangulation.) We show that any $n$-gon has a pseudo-convex decomposition of size $\lceil n / 2\rceil-1$.

Note that any quadrangulation (a decomposition into quadrilaterals) of an $n$-gon is a pseudo-convex decomposition, and it also has $\lceil n / 2\rceil-1$ faces. However, not every polygon has a quadrangulation. Allowing Steiner points on the boundary of the polygon, Ramaswami, Ramos, and Toussaint [30] show that the minimum quadrangulation of every $n$-gon has at most $\lfloor 2 n / 3\rfloor+O(1)$ faces in the worst case.

\section{Basic Combinatorial Properties}

Our first (trivial) observation is that $\psi_{d}(n) \leq \kappa_{d}(n), \psi_{p}(n) \leq \kappa_{p}(n)$, and $\psi_{c}(n) \leq$ $\kappa_{c}(n)$. It is well known that $\kappa_{c}(n) \leq \kappa_{p}(n) \leq \kappa_{d}(n)$. For pseudo-convex faces we trivially have $\psi_{c}(n) \leq \psi_{p}(n) . \psi_{p}(n) \leq \psi_{d}(n)$ follows from the bounds given in the previous section.

Next we observe that $\psi_{d}(n+1) \leq \psi_{d}(n)+1, \psi_{p}(n+1) \leq \psi_{p}(n)+1$, and $\psi_{c}(n+1) \leq \psi_{c}(n)+1$. This follows by induction when inserting the points sorted 

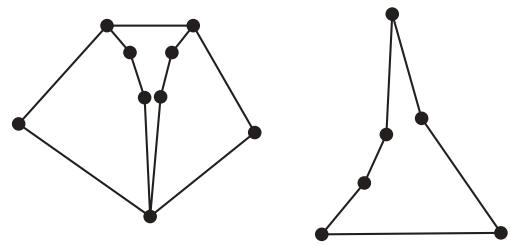

Fig. 2. Sets with non-monotone behavior

according to their $x$-coordinates. For covering and partitioning, the last inserted vertex is a singleton (hence convex); for decomposing, the difference between the convex hull of the first $n$ points and all $n+1$ points is a pseudo-triangle with a corner at the last inserted point.

The following lemma establishes an interesting connection between the convex partition number and the pseudo-convex decomposition number.

Lemma 1. For every finite point set $S$, we have $\psi_{d}(S) \leq 3 \kappa_{p}(S)-2$ and thus $\psi_{d}(n) \leq$ $3 \kappa_{p}(n)-2$.

Proof. Every pointed pseudo-triangulation of $S$ is a pseudo-convex decomposition of $S$ with $n-2$ faces. We construct a pseudo-convex decomposition as follows: Take the $\kappa_{p}(S)$ polygonal domains of a minimum convex partition of $S$ (some of which may be singletons or line segments) and pseudo-triangulate their complement in a pointed way. By triangulating each convex face, we can transform this pseudo-convex decomposition into a pseudo-triangulation: this shows that we use fewer than $n-2$ faces. For a convex face of size $k_{i} \geq 3$, this transformation creates $k_{i}-3$ additional faces.

Since each point of $S$ is the vertex of exactly one face of a convex partition, we have $\sum_{i=1}^{\kappa_{p}(S)} k_{i}=n$, and so we can reduce the number of faces by at least $\sum_{i=1}^{\kappa_{p}(S)}\left(k_{i}-\right.$ $3)=n-3 \kappa_{p}(S)$. Therefore, a minimum convex partition of $S$ directly yields a pseudo-convex decomposition of $S$ with at most $(n-2)-\left(n-3 \kappa_{p}(S)\right)=3 \kappa_{p}(S)-2$ faces.

The pseudo-convex decomposition, partition, and covering numbers for a particular point set $S$ are not necessarily monotone. Consider the examples in Fig. 2. On the left, a set $S$ with 9 points and $\psi_{d}(S)=3$. Removing the bottom-most point of $S$ results in a set $S^{\prime}$ with 8 points and $\psi_{d}\left(S^{\prime}\right)=4$. On the right, a set $S$ with 6 points and $\psi_{c}(S)=\psi_{p}(S)=1$. Removing the top-most point of $S$ results in a set $S^{\prime}$ with 5 points and $\psi_{c}\left(S^{\prime}\right)=\psi_{p}\left(S^{\prime}\right)=2$.

\section{Pseudo-Convex Decompositions}

We first give a formula for the number of faces in a pseudo-convex decomposition:

Lemma 2. Let $S$ be a set of $n$ points in general position. Let $P$ be a pseudo-convex decomposition of $S, n_{k}$ the number of convex $k$-gons in $P$, and $p$ the number of pointed 
vertices. Then the number of faces of $P$ is

$$
|P|=2 n-p-2-\sum_{k=4}^{n} n_{k}(k-3) .
$$

Proof. We triangulate every convex $k$-gon in our decomposition (for $k \geq 4$ ) and thus obtain a pseudo-triangulation with $2 n-p-2$ pseudo-triangles [16]. Triangulating a convex $k$-gon introduces $k-3$ new faces. The proof follows.

Corollary 1. The number of faces in a pointed pseudo-convex decomposition is

$$
|P|=n-2-\sum_{k=4}^{n} n_{k}(k-3) .
$$

Although the pseudo-convex decomposition number for a particular point set $S$ might not be monotone (recall Fig. 2), $\psi_{d}(n)$ nevertheless increases monotonically with $n$.

Theorem 1. The pseudo-convex decomposition number increases monotonically with the number of points.

Proof. We want to show that $\psi_{d}(n) \leq \psi_{d}(n+1)$, which is equivalent to showing that for every point set $S$ with $|S|=n, \psi_{d}(S) \leq \psi_{d}(n+1)$ holds. Consider a set $S$ of $n$ points and let $q \in S$ be a point on the convex hull of $S$. We place a new vertex $q^{+}$ arbitrarily close to $q$ to get the set $S^{+}=S \cup q^{+}$such that both $q$ and $q^{+}$lie on the convex hull of $S^{+}$. Note that $S^{+} \backslash q$ has the same order type as $S$, that is, for any two points $p_{1}, p_{2} \in S \backslash q$ the triples $p_{1}, p_{2}, q$ and $p_{1}, p_{2}, q^{+}$have the same orientation.

As $S^{+}$has $n+1$ points, it has a pseudo-convex decomposition $D^{+}$with at most $\psi_{d}(n+1)$ faces. Note that the face $F$ in $D^{+}$which contains the edge $q q^{+}$has to be convex, otherwise $P$ would be a pseudo-triangle and the points $q$ and $q^{+}$would lie on opposite sides of the line through one of the edges $F$. Now contract the edge $q q^{+}$into $q$. By this transformation the face $F$ loses one edge, but all other faces of $\mathrm{D}^{+}$remain combinatorially unchanged, that is, either convex polygons or valid pseudo-triangles. Thus we obtain a pseudo-decomposition $D$ of $S$ which has either the same number of faces as $D^{+}$or, in the case that $F$ was a triangle, one fewer. Therefore $\psi_{d}(S) \leq \psi_{d}\left(S^{+}\right) \leq \psi_{d}(n+1)$.

\subsection{Two Geometric Ramsey-type Results}

Let $S$ be a planar point set in general position. We say that an empty $k$-gon is a simple polygon spanned by $k$ points of $S$ that contains no point of $S$ in its interior.

Theorem 2. Every set of 8 points in general position contains either an empty convex pentagon or two disjoint empty convex quadrilaterals. 


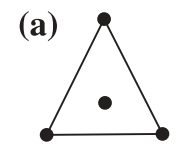

$n=4$

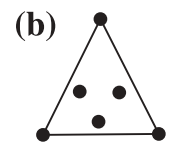

$n=6$ (c)

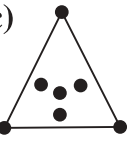

$n=7$

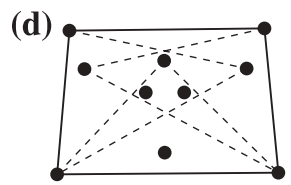

$n=10$ (e)

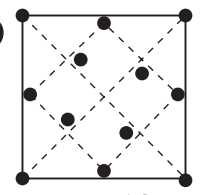

$n=12$ (f)

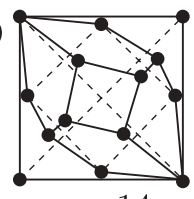

$n=14$

Fig. 3. (a)-(e) Lower bound examples, (f) every minimum decomposition is non-pointed

Theorem 3. Every set of 11 points in general position contains either an empty convex hexagon or an empty convex pentagon and a disjoint empty convex quadrilateral.

Both results were established with the help of the order type data base [2, 3]. In Subsection 3.4 we also provide an intuitive geometric proof of Theorem 2 that requires only a moderate number of case distinctions.

\subsection{Small Point Sets}

In this section we give tight upper and lower bounds on $\psi_{d}(n)$ for sets of up to 13 points. Recall that $\psi_{d}(n+1) \leq \psi_{d}(n)+1$ and (by Theorem 1$) \psi_{d}(n) \leq \psi_{d}(n+1)$. Obviously $\psi_{d}(3)=1$. If four points do not lie in convex position (see Fig. 3(a)) then every decomposition needs at least two faces and hence $\psi_{d}(4)=2$ and $\psi_{d}(5) \geq 2$. Every set of 5 points contains an empty convex quadrilateral [8]. Pseudo-triangulating in a pointed way around this quadrilateral yields $\psi_{d}(5)=2$ by Corollary 1 .

$\psi_{d}(5)=2$ implies $\psi_{d}(6) \leq 3$. Figure $3($ b) shows a configuration $S$ of 6 points such that every pseudo-convex decomposition of $S$ has at least 3 faces. $S$ does not span any empty convex $k$-gon for $k>4$. Every empty convex quadrilateral spanned by $S$ necessarily uses all three inner points, so every partition of $S$ can contain at most one convex quadrilateral. Then Corollary 1 implies $\psi_{d}(6)=6-2-(4-3)=3$ for pointed pseudo-decompositions, which are optimal in this case.

$\psi_{d}(6)=3$ implies $\psi_{d}(7) \leq 4$. Figure 3(c) shows a configuration $S$ of 7 points such that every pseudo-convex decomposition of $S$ has at least 4 faces. The argument is similar to the one for the example with 6 points. Again, $S$ does not span any empty convex $k$-gon for $k>4$. Every pointed decomposition contains at most one convex quadrilateral, because every convex quadrilateral contains the point in the center. Every additional quadrilateral forces at least one additional vertex to be non-pointed, so a non-pointed decomposition cannot contain fewer faces than a pointed one. Therefore, $\psi_{d}(7)=7-2-(4-3)=4$.

$\psi_{d}(7)=4$ implies $\psi_{d}(8) \geq 4$. Theorem 2 together with Corollary 1 implies $\psi_{d}(8) \leq 8-2-2=4$. We construct this decomposition by pseudo-triangulating in a pointed way the complement of the convex polygon(s) guaranteed by Theorem 2 .

Every set of 10 points contains an empty pentagon [17] and so Corollary 1 implies $\psi_{d}(10) \leq 10-2-(5-3)=6$. Figure $3(\mathrm{~d})$ (which is a close relative of a 
construction in [18]) shows a configuration $S$ of 10 points such that every pseudoconvex decomposition of $S$ has at least 6 faces. First note that $S$ does not span any empty convex pentagon and a disjoint empty convex quadrilateral. Furthermore, every empty convex pentagon spanned by $S$ necessarily contains the three points in the upper center, so every partition of $S$ can contain at most one convex pentagon. If we start our decomposition with a pentagon, then we can not add a quadrilateral without creating at least one non-pointed vertex. Therefore, a nonpointed decomposition cannot have fewer faces than a pointed one, which implies $\psi_{d}(10)=10-2-(5-3)=6$.

$\psi_{d}(10)=6$ implies that $\psi_{d}(9) \geq 5$. Since every set of 9 points contains two disjoint empty convex quadrilaterals [18], we have (with Corollary 1) $\psi_{d}(9) \leq 9-$ $2-2 \cdot(4-3)=5$.

$\psi_{d}(10)=6$ also implies $\psi_{d}(11) \geq 6$. Theorem 3 together with Corollary 1 yields $\psi_{d}(11) \leq 11-2-3=6$. We construct this decomposition by pseudo-triangulating in a pointed way around the convex polygon(s) guaranteed by Theorem 3 .

$\psi_{d}(11)=6$ implies $\psi_{d}(12) \leq 7$. Figure $3(\mathrm{~d})$ shows a configuration $S$ of 12 points such that every pseudo-convex decomposition of $S$ has at least 7 faces. The largest empty convex set in this configuration is a hexagon. Every empty convex pentagon or hexagon contains at least three of the four inner points and thus separates the other points, so that no disjoint convex quadrilateral can be found. The coordinates of this point set are: $(0,0),(0,20),(20,20),(20,0),(1,10),(10,19),(19,10),(10,1)$, $(5,7),(7,15),(15,13),(13,5)$.

$\psi_{d}(12)=7$ implies $\psi_{d}(13) \leq 8$. The point set with the following coordinates requires 8 faces for every pseudo-convex decomposition: $(65535,65535),(0,0)$, (29293, 36890), (15166, 26472), (27461, 37283), (32929, 42217), (29439, 42711), (27746, 42587), (27491, 42925), (32135, 45720), (29447, 45175), (31736, 48764), (19257, 42830). This lower bound example was found with the help of the order type database [3].

Non-pointed Decompositions. All upper bounds on $\psi_{d}(n)$ for $n \leq 13$ can be achieved with pointed decompositions as described in the preceding paragraphs. Also the general upper bound can be realized with a pointed decomposition, as we will see in the next subsection. However, for $n \geq 10$, there are point sets such that an optimal (minimal) decomposition is always non-pointed. See, for example, Fig. 3(e) which shows a configuration of 14 points such that every minimal pseudo-convex decomposition is non-pointed. The coordinates for this point set are the same as the ones for Fig. 3(d) with the addition of $(4,5)$ and $(16,15)$.

\subsection{Upper Bound}

Our upper bound construction is based on exact pseudo-convex decomposition numbers for small point sets. Assume that we are given a set $S$ with $n$ points and that we know the value of $\psi_{d}(\cdot)$ up to some $k<n$. We choose a point $p$ on the convex hull of $S$. Now we partition the plane by half-lines emanating from $p$ into $\lceil(n-1) /(k-1)\rceil$ wedges such that all but at most one wedge contains exactly $k-1$ points of $S \backslash\{p\}$ (one of the wedges contains $n-1-\left\lfloor\frac{n-1}{k-1}\right\rfloor(k-1)$ points of $S \backslash\{p\}$ ). Let a petal be the convex hull of points in a wedge together with $p$. 


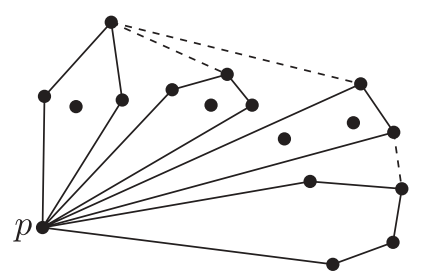

Fig. 4. Petals of size 5

We have a total of $\lceil(n-1) /(k-1)\rceil$ petals, each petal of $k^{\prime} \leq k$ points can be decomposed into at most $\psi_{d}\left(k^{\prime}\right)$ faces. Two adjacent petals can be combined with a pseudo-triangle into one larger convex set. We combine inductively adjacent convex sets (all including $p$ ) until we obtain the convex hull of $S$. We have proved an upper bound of

$$
\begin{aligned}
\psi_{d}(n) & \leq\left\lfloor\frac{n-1}{k-1}\right\rfloor \psi_{d}(k)+\psi_{d}\left(n-\left\lfloor\frac{n-1}{k-1}\right\rfloor(k-1)\right)+\left\lceil\frac{n-1}{k-1}\right\rceil-1, \\
& \leq\left\lfloor\frac{n-1}{k-1}\right\rfloor\left(\psi_{d}(k)+1\right)+\psi_{d}\left(k_{n}^{\prime}\right)=\frac{\psi_{d}(k)+1}{k-1} n+O(1),
\end{aligned}
$$

where $k_{n}^{\prime}=n-\left\lfloor\frac{n-1}{k-1}\right\rfloor(k-1)$. The currently known minimal value of $\left(\psi_{d}(k)+1\right) /$ $(k-1)$ is attained for $k=11$ and $\psi_{d}(11)=6$. Taking into account the term $\psi_{d}\left(k_{n}^{\prime}\right)$, $k_{n}^{\prime}<k$, in Inequality (1), we obtain a general upper bound of

$$
\psi_{d}(n) \leq \frac{\psi_{d}(11)+1}{11-1} n=\frac{7 n}{10} .
$$

For some small values of $n$, Inequality (1) with another $k$ may give a better upper bound: For instance, we have $\psi_{d}(15) \leq 9$ for $k=8$, completing the first row of Table 1.

\subsection{Proof of Theorem 2}

Let $S$ be a set of $n$ points in general position in the plane. Recall that an empty $k$-gon is a polygon spanned by $k$ points in $S$ whose interior does not contain any points of $S$. Let $H \subset S$ be the set of points on the convex hull of $S$. We call the points of $H$ the outer points and the points of $I=S \backslash H$ the inner points of $S$. In this section we prove Theorem 2, which we restate here for completeness.

Theorem 2. Every set of 8 points in general position contains either an empty convex pentagon or two disjoint empty convex quadrilaterals.

The proof of Theorem 2 consists of a case distinction based on the number of outer points.

Lemma 3. If $|H| \geq 6$ then $S$ contains an empty convex pentagon. 
Proof. If $|H|=8$, then $S$ contains an empty convex octagon.

If $|H|=7$, then there is a unique point $x \in I$. Choose an arbitrary $p \in H$. There are at least three point of $H$ on one side of the line through $p$ and $x$. Together with these three points, $p$ and $x$ form an empty convex pentagon.

If $|H|=6$, then $I$ consists of exactly two points $x$ and $y$. They span a line that has at least three points of $H$ on one side. Together with these 3 points, $x$ and $y$ form a convex pentagon.

Lemma 3 implies Theorem 2 for $|H| \geq 6$. To prove Theorem 2 for $|H| \leq 5$ we first collect several useful observations.

Observations and Definitions. We denote the convex hull of the inner points $I$ of $S$ by $P=\mathcal{C} H(I)$. Let $H^{\prime}$ be the vertex set of $P$. Two adjacent vertices $q, r$ of $P$ form a face $f$ of $P$, denoted by $f=\{q, r\}$. We say that a point $p \in H$ sees a face $f$ of $P$ or that $f$ is visible to $p$ if $p$ and $P$ are on different sides of the line $l_{f}$ spanned by the vertices of $f$. If $p$ sees $f$ then we call the pair $(p, f)$ a visibility pair. Let $V(p)$ be the set of faces visible to $p$, and let $V P$ be the total number of visibility pairs, that is, $V P=\sum_{p \in H}|V(p)|$.

Lemma 4. If $|H| \leq 5$, then (a) for every $p \in H$, we have $1 \leq|V(p)| \leq\left|H^{\prime}\right|-1$; and (b) every face $f$ of $P$ is visible to at least one vertex $p \in H$.

Proof. (a) Consider a point $p \in H$ and draw two tangents from $p$ to $P$. By the general position assumption, there are two well-defined points $q_{1}, q_{2} \in H^{\prime}, q_{1} \neq q_{2}$, incident to these two tangents. If $|I| \geq 3$, then the convex hull $P=\mathcal{C} H(I)$ is a closed polygonal curve; and the points $q_{1}$ and $q_{2}$ split $P$ into two non-empty open polygonal curves. Point $p$ sees every face of $P$ along the polygonal curve closer to $p$, and it does not see any faces along the other polygonal curve.

(b) Consider a face $f$ of $P$, and let $h(f)$ be the open halfplane bounded by the line through $f$ that does not contain $P$. There must be an outer point in $h(f)$, otherwise the endpoints of $f$ would be outer points. By definition, every point $p \in H \cap h(f)$ sees $f$.

Observation 1. The set $\{p \in H \mid p$ sees $f\} \cup f$ forms an empty convex polygon for every face $f$ of $P$.

Observation 1 immediately implies:

Observation 2. If there are three or more vertices of $H$ that see the same face $f$ of $P$, then $S$ contains an empty convex pentagon. (See Fig. 5, left.)

Observation 2 and the pigeonhole principle imply:

Observation 3. If $V P>2 \cdot\left|H^{\prime}\right|$, then $S$ contains an empty convex pentagon. 

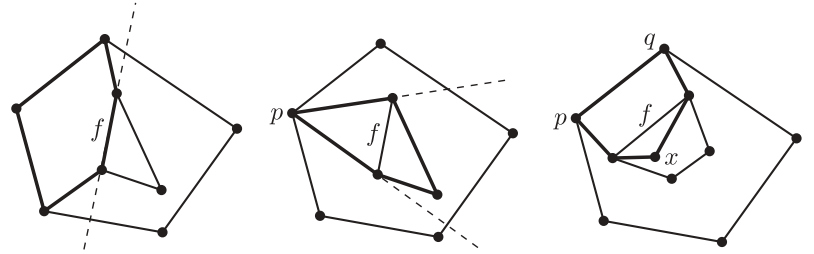

Fig. 5. Observation 2 (left), Observation 4 (middle), and Observation 6 (right)

Next, we collect some properties of the outer vertices that see only one face of the inner polygon, that is, for every $p \in H$ such that $|V(p)|=1$.

Observation 4. $\{p \in H \mid V(p)=\{f\}\} \cup H^{\prime}$ forms a convex polygon for every face $f$ of P. (See Fig. 5, middle.)

Observation 4 implies:

Observation 5. If $I \backslash H^{\prime}=\emptyset$, then $\{p \in H \mid V(p)=\{f\}\} \cup H^{\prime}$ forms an empty convex polygon for every face $f$ of $P$.

Observation 6. If $I \backslash H^{\prime} \neq \emptyset$, then there is an inner point $x \in I \backslash H^{\prime}$ such that $\{p \in H \mid V(p)=\{f\}\} \cup f \cup\{x\}$ forms an empty convex polygon for every face $f$ of P. (See Fig. 5, right.)

If $\left|H^{\prime}\right| \geq 3$ then Observation 5 and Observation 6 jointly imply:

Observation 7. If $\left|H^{\prime}\right| \geq 3$ and if there are two points $p$ and $q$ in $H$ such that $V(p)=$ $V(q)=\{f\}$ for some face $f$ of $P$, then $S$ contains an empty convex pentagon.

And again by the pigeonhole principle:

Observation 8. If $\left|H^{\prime}\right| \geq 3$ and $|\{p \in H|| V(p) \mid=1\}|>\left|H^{\prime}\right|$, then $S$ contains an empty convex pentagon.

Now assume that there is an outer vertex $p \in H$ that sees two consecutive faces $f_{1}$ and $f_{2}$ of $P$; and let $p^{\prime}=f_{1} \cap f_{2} \in H^{\prime}$. The line $l_{p, p^{\prime}}$ through $p$ and $p^{\prime}$ partitions the plane into two halfplanes. One of them contains $f_{1}$, the other $f_{2}$. We call them $h_{f_{1}}\left(l_{p, p^{\prime}}\right)$ and $h_{f_{2}}\left(l_{p, p^{\prime}}\right)$, respectively (Fig. 6, left.).

Observation 9. (a) If $p, q \in H, f=\left\{p^{\prime}, q^{\prime}\right\} \in V(p) \cap V(q)$, and there is an inner point in $h_{f}\left(l_{p, p^{\prime}}\right) \cap h_{f}\left(l_{q, q^{\prime}}\right) \cap P$, then $p, q, p^{\prime}, q^{\prime}$ and the inner point form an empty convex pentagon (Fig. 6, middle). 

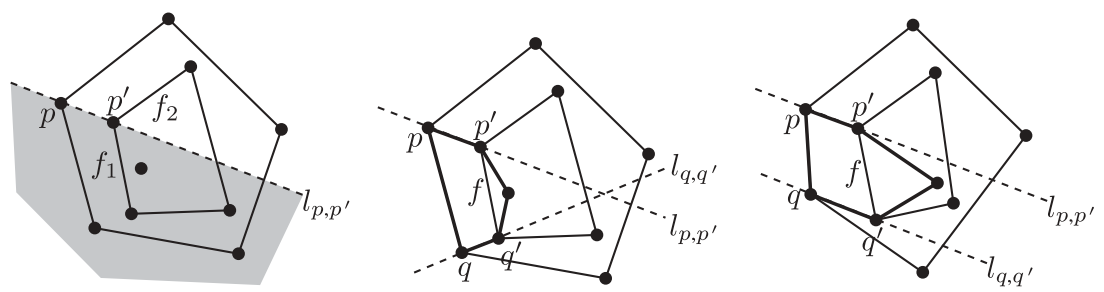

Fig. 6. $h_{f_{1}}\left(l_{p, p^{\prime}}\right)$ (left), Observation 9(a) (middle), and Observation 9(b) (right)
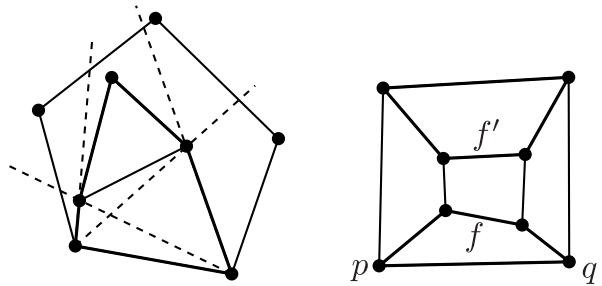

Fig. 7. $|H|=5$ (left). $|H|=4$ and $\left|H^{\prime}\right|=4$ (right)

(b) If $p \in H, f=\left\{p^{\prime}, q^{\prime}\right\} \in V(p)$, there is an inner point contained in $h_{f}\left(l_{p, p^{\prime}}\right)$, and $q \in H$ such that $V(q)=\{f\}$, then $S$ contains an empty convex pentagon (Fig. 6 , right).

Note that we assume that the cyclic orientation of the convex hull of both $H$ and $H^{\prime}$ are the same (say, counter-clockwise), as indicated in Fig. 6.

Proof of Theorem 2 (for $|H| \leq 5$ ). We now continue the case distinction for the proof of Theorem 2 based on the number of points in $H$ and in $H^{\prime}$.

Case $|H|=5$. $P$ necessarily is a triangle and every inner point belongs to $H^{\prime}$, that is, $\left|H^{\prime}\right|=3$. Lemma 4 implies that $|V(p)| \leq 2$ for every $p \in H$.

If there are at least two outer vertices that can see two faces of $P$, then $V P \geq$ $2 \cdot 2+3=7>6=2 \cdot\left|H^{\prime}\right|$; and by Observation $3, S$ contains an empty convex pentagon.

Otherwise, there is at most one outer vertex that sees two faces, so four or more outer vertices can each see only one face of $P$. Since $P$ has only three faces, the number of vertices $p$ for which $|V(p)|=1$ is greater than the number of faces of $P$, and so by Observation $8, S$ contains an empty convex pentagon (see Fig. 7, left).

Case $|H|=4$ and $\left|H^{\prime}\right|=4$. Since $\left|H^{\prime}\right|=4$, every inner point belongs to $H^{\prime}$, that is, $I \backslash H^{\prime}=\emptyset$. Lemma 4 implies that $|V(p)| \leq 3$ for every $p \in H$.

If there is an outer vertex $p$ such that $|V(p)|=1$ then, by Observation $5, p$ forms an empty convex pentagon together with $H^{\prime}$. Therefore we can assume that $|V(p)| \geq 2$ for every $p \in H$. If there is a $p \in H$ such that $|V(p)|=3$, then $V P \geq 3+3 \cdot 2=9>8=2 \cdot\left|H^{\prime}\right|$, so by Observation $3, S$ contains an empty convex pentagon. Thus we can assume that $|V(p)|=2$ for every $p \in H$. 

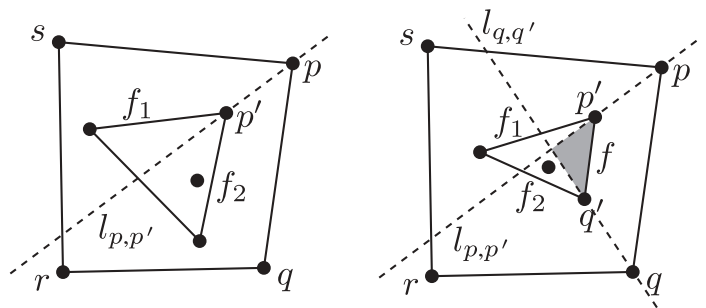

Fig. 8. $|H|=4$ and $\left|H^{\prime}\right|=3, h_{f}\left(l_{p, p^{\prime}}\right) \cap h_{f}\left(l_{q, q^{\prime}}\right) \cap P$ is shaded in the right figure

If there is a face $f$ of $P$ that is visible to more than 2 vertices then, by Observation $2, S$ contains an empty convex pentagon. So we can further assume that every face $f$ of $P$ is visible to exactly 2 outer vertices.

Let $f$ be a face of $P$, and let $p, q$ be the outer vertices that see $f$. By Observation $1, p, q$ and the vertices of $f$ form an empty convex quadrilateral. The opposite face $f^{\prime}=H^{\prime} \backslash f$ is also visible to two vertices, but not to $p$ or $q$, because each of them sees only two faces. Again by Observation 1, the remaining two outer vertices form a second quadrilateral together with the vertices of $f^{\prime}$ (see Fig. 7, right).

Case $|H|=4$ and $\left|H^{\prime}\right|=3$. Since $\left|H^{\prime}\right|=3$, there is one inner point $x$ that does not belong to $H^{\prime}$, that is, $I \backslash H^{\prime}=\{x\}$. Lemma 4 implies that $|V(p)| \leq 2$ for every $p \in H$. We distinguish subcases according to the number of outer vertices that see two faces of $P$.

If there are more than two outer vertices that see two faces of $P$, then $V P \geq$ $3 \cdot 2+1=7>6=2 \cdot\left|H^{\prime}\right|$ and by Observation $3, S$ contains an empty convex pentagon. If, on the other hand, $|V(p)|=1$ for every $p \in H$, then, by the pigeonhole principle, one face $f$ of $P$ must be visible to two vertices, and by Observation 7 , $S$ contains an empty convex pentagon. We can, therefore, assume that either exactly one or exactly two outer vertices see two faces of $P$ and that each of the remaining outer vertices see exactly one face of $P$. Furthermore, we can assume by Observation 7 that if two outer vertices each see only one face of $P$ then these two faces are different.

$|V(p)|=2$ for exactly one outer vertex $p$, see Fig. 8 (left).

Let $V(p)=\left\{f_{1}, f_{2}\right\}$. According to our assumptions, there are two outer vertices $s$ and $q$ such that $V(s)=\left\{f_{1}\right\}$ and $V(q)=\left\{f_{2}\right\}$. Since $x$ must be contained in either $h_{f_{1}}\left(l_{p, p^{\prime}}\right)$ or $h_{f_{2}}\left(l_{p, p^{\prime}}\right)$, we can apply Observation 9 (b) to either $p, f_{1}$, and $s$ or $p, f_{2}$, and $q$ and hence $S$ contains an empty convex pentagon.

$|V(p)|=|V(q)|=2$ for exactly two outer vertices $p$ and $q$, see Fig. 8 (right).

$\left|H^{\prime}\right|=3$ implies that $V(p) \cap V(q) \neq \emptyset$. If $V(p)=V(q)$ then necessarily at least one outer vertex $r \neq p, q$ sees a face from $V(p)$ and hence Observation 2 implies that $S$ contains an empty convex pentagon.

Let therefore $V(p)=\left\{f, f_{1}\right\}$ and $V(q)=\left\{f, f_{2}\right\}$ with $f_{1} \neq f_{2}$. If one of the remaining two outer vertices $s$ and $r$ sees $f$ then Observation 2 again implies that $S$ contains an empty convex pentagon. We can therefore assume that $V(s)=\left\{f_{1}\right\}$ and $V(r)=\left\{f_{2}\right\}$. If $x$ is contained in $h_{f}\left(l_{p, p^{\prime}}\right) \cap h_{f}\left(l_{q, q^{\prime}}\right) \cap P$ then 

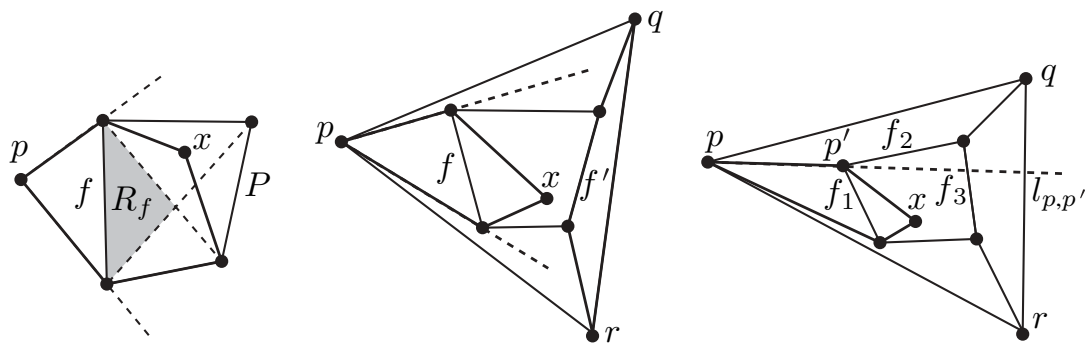

Fig. 9. Observation 10 (left), Observation 11 (middle), and Observation 12 (right)

Observation 9(a) implies that $S$ contains an empty convex pentagon. Otherwise $x$ has to be contained in $h_{f_{1}}\left(l_{p, p^{\prime}}\right)$ or $h_{f_{2}}\left(l_{q, q^{\prime}}\right)$. We can apply Observation 9(b) to either $p, f_{1}$, and $s$ or $q, f_{2}$, and $r$ and hence $S$ contains an empty convex pentagon.

Cases $|H|=3$. There are $|I|=5$ inner points. If $\left|H^{\prime}\right|=5$ then the inner points form an empty convex pentagon. The remaining two cases are $\left|H^{\prime}\right|=4$ and $\left|H^{\prime}\right|=3$.

Case $|H|=3$ and $\left|H^{\prime}\right|=4$. Since $\left|H^{\prime}\right|=4$, there is one inner point $x$ that does not belong to $H^{\prime}$, that is, $I \backslash H^{\prime}=\{x\}$. The diagonals of the inner quadrilateral $P$ partition $P$ into 4 regions. Each of them contains exactly one face of $P$. Let $R_{f}$ be the region containing face $f$. Since we assume all points to be in general position, $x$ is contained in exactly one of these regions. Before we begin with a detailed case analysis we collect some additional observations.

Observation 10. If $V(p)=\{f\}$ for $p \in H$ and $x \notin R_{f}$, then $S$ contains an empty convex pentagon (Fig. 9, left).

Observation 1 and Observation 6 imply:

Observation 11. If $V(p)=\{f\}$ for $p \in H$ and the opposite face $f^{\prime}=H^{\prime} \backslash f \in$ $V(q) \cap V(r)$ for $q \neq r \in H \backslash\{p\}$, then $S$ contains two disjoint empty convex quadrilaterals (Fig. 9, middle).

Observation 12. If $V(p)=\left\{f_{1}, f_{2}\right\}$ for $p \in H$ with $f_{1} \cap f_{2}=p^{\prime}, x \in h_{f_{1}}\left(l_{p, p^{\prime}}\right)$ and $f_{3}=H^{\prime} \backslash f_{1} \in V(q) \cap V(r)$ for $q \neq r \in H \backslash\{p\}$, then $S$ contains two empty convex quadrilaterals (Fig. 9, right).

Recall from Lemma 4 that every face $f$ of $P$ is visible to at least one vertex from $H$. Since $|H|=3$, at least one of the outer vertices needs to see at least two faces. We distinguish the following subcases:

$|V(p)|=1$ for at least one outer vertex $p$.

Let $V(p)=\{f\}$. If there is a second outer vertex $q$ such that $V(q)=\{f\}$, then by Observation $7 S$ contains an empty convex pentagon. If there is a second 
(a)

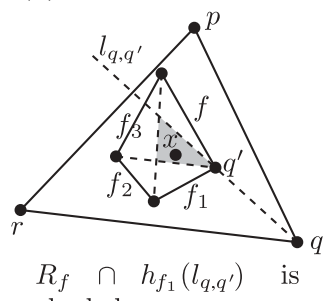

shaded. (b)

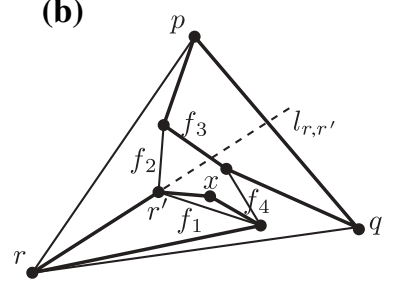

(c)

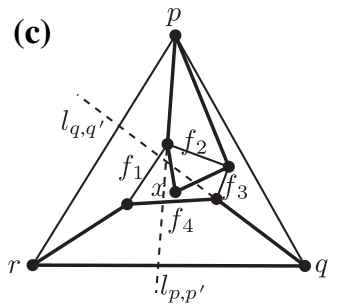

Fig. 10. $|H|=3,\left|H^{\prime}\right|=4$

outer vertex $q$ such that $V(q)=\left\{f^{\prime}\right\}$, where $f^{\prime} \neq f$, Observation 10 can be applied to at least one of $p$ and $q$, because $x$ is contained in at most one of $R_{f}$ and $R_{f^{\prime}}$ and hence $S$ contains an empty convex pentagon.

We can therefore assume that the remaining outer vertices $q$ and $r$ both see at least two faces of $P$. We cannot have both $f \in V(q)$ and $f \in V(r)$, since $f \subset \mathcal{C H}(I)$ cannot be visible to all vertices of $H$. Let $f_{2}=H^{\prime} \backslash f$ denote the opposite face of $f$. If $\left\{f_{2}\right\} \in V(q) \cap V(r)$ then Observation 11 implies that $S$ contains two disjoint empty convex quadrilaterals.

We can therefore assume w.l.o.g. that $\left\{f, f_{1}\right\} \subseteq V(q), f_{2} \notin V(q),\left\{f_{2}, f_{3}\right\} \subseteq$ $V(r)$, and $f \notin V(r)$ where $f_{1}$ and $f_{3}$ denote the two remaining, opposite faces of $P$ (see Fig. 10(a)). Now consider the line $l_{q, q^{\prime}}$ with $q^{\prime}=f \cap f_{1}$. If $x \in h_{f}\left(l_{q, q^{\prime}}\right)$ then Observation 9(b) implies that $S$ contains an empty convex pentagon. So we can assume that $x \in h_{f_{1}}\left(l_{q, q^{\prime}}\right)$. If $x \notin R_{f}$ then Observation 10 implies that $S$ contains an empty convex pentagon. So we can also assume that $x \in R_{f}$. Since now $x \in h_{f_{1}}\left(l_{q, q^{\prime}}\right)$ and $x \in R_{f}$, that is, $R_{f} \cap h_{f_{1}}\left(l_{q, q^{\prime}}\right) \neq \emptyset$, the two endpoints of $f_{2}$ must lie in $h_{f_{1}}\left(l_{q, q^{\prime}}\right)$ as well. They form an empty convex pentagon together with $x$ and the points $q, q^{\prime}$.

From now on we can assume that $|V(p)| \geq 2$ for every $p \in H$. Lemma 4 implies that $|V(p)| \leq 3$ for every $p \in H$. If $|V(p)|=3$ for every $p \in H$ then $V P=3 \cdot 3=9>8=2 \cdot\left|H^{\prime}\right|$, so by Observation $3, S$ contains an empty convex pentagon. Thus we can assume that $|V(p)|=2$ for at least one $p \in H$. By Observation 2 we can also assume that no face $f$ of $P$ is seen by all outer vertices.

$V(p)=V(q)$ for two outer vertices $p$ and $q$. Let $r$ denote the third outer vertex. Since $|V(p)| \geq 2$ for every $p \in H$, we necessarily have $|V(p)|=|V(q)|=$ $|V(r)|=2$. Furthermore, $r$ sees exactly the two faces of $P$ that $p$ and $q$ do not see. Let $V(r)=\left\{f_{1}, f_{2}\right\}$ with $f_{1} \cap f_{2}=r^{\prime}$ and $V(p)=V(q)=\left\{f_{3}, f_{4}\right\}$ (see Fig. 10(b)). Now consider the line $l_{r, r^{\prime}} x$ must lie either in $h_{f_{1}}\left(l_{r, r^{\prime}}\right)$ or in $h_{f_{2}}\left(l_{r, r^{\prime}}\right)$. Since both $H^{\prime} \backslash f_{1}$ and $H^{\prime} \backslash f_{2}$ are contained in $V(p) \cap V(q)$, Observation 12 implies in either case that $S$ contains two empty convex quadrilaterals.

$V(p) \cap V(q)=\emptyset$ for two outer vertices $p$ and $q$. Let $r$ denote the third outer vertex. If either $V(p)=V(r)$ or $V(q)=V(r)$ then the previous case applies. So we can assume that $V(p) \neq V(r) \neq V(q)$. Necessarily $|V(p)|=|V(q)|=2$ and $|V(r)| \in\{2,3\}$. Let $V(p)=\left\{f_{1}, f_{2}\right\}$ with $p^{\prime}=f_{1} \cap f_{2}$ and $V(q)=\left\{f_{3}, f_{4}\right\}$ with 

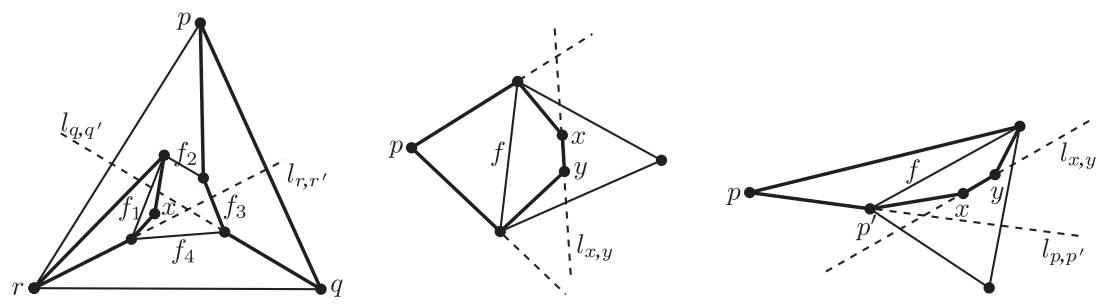

Fig. 11. $|H|=3,\left|H^{\prime}\right|=4$ (left). Observation 13 (middle and right)

$q^{\prime}=f_{3} \cap f_{4}$. There must be two faces $f \in V(r) \cap V(p)$ and $f^{\prime} \in V(r) \cap V(q)$ with $f^{\prime} \neq f$. W.1.o.g. $\left\{f_{1}, f_{4}\right\} \subseteq V(r)$ with $r^{\prime}=f_{1} \cap f_{4}$ (see Fig. 10(c)).

Since we assume all points to be in general position, we know that $x$ must lie either in $h_{f_{1}}\left(l_{r, r^{\prime}}\right)$ or in $h_{f_{4}}\left(l_{r, r^{\prime}}\right)$. If $x \in h_{f_{1}}\left(l_{p, p^{\prime}}\right) \cap h_{f_{4}}\left(l_{q, q^{\prime}}\right)$ then Observation 9(a) applies to either $p$ and $r$ or $q$ and $r$ and implies that $S$ contains an empty convex pentagon.

Let us assume that $x \notin h_{f_{1}}\left(l_{p, p^{\prime}}\right)$ which is equivalent to $x \in h_{f_{2}}\left(l_{p, p^{\prime}}\right)$. Since $f_{4}=H^{\prime} \backslash f_{2}$ is contained in $V(q) \cap V(r)$, Observation 12 implies that $S$ contains two empty convex quadrilaterals. Symmetrically, if $x \notin h_{f_{4}}\left(l_{q, q^{\prime}}\right)$ then necessarily $x \in h_{f_{3}}\left(l_{q, q^{\prime}}\right)$. Since $f_{1}=H^{\prime} \backslash f_{3}$ is contained in $V(p) \cap V(r)$, Observation 12 again implies that $S$ contains two empty convex quadrilaterals.

$V(p) \neq V(q)$ and $V(p) \cap V(q) \neq \varnothing$ for any two outer vertices $p$ and $q$. Let $p, q$, and $r$ denote the outer vertices. The condition above implies that one of them has to see 3 faces. W.1.o.g. let us assume that $|V(p)|=3$. We also know that one of them sees only two faces. Again w.l.o.g. let us assume that $|V(q)|=2$. $V(q)$ can not be a subset of $V(p)$ since that would imply that $V(q)$ and $V(r)$ are disjoint. So let $V(p)=\left\{f_{1}, f_{2}, f_{3}\right\}$ and $V(q)=\left\{f_{3}, f_{4}\right\}$ with $q^{\prime}=f_{3} \cap f_{4}$. Necessarily $\left\{f_{4}, f_{1}\right\} \in V(r)$ and $f_{3} \notin V(r)$.

If $f_{2} \in V(r)$ then consider the line $l_{q, q^{\prime}} \cdot x$ must lie either in $h_{f_{3}}\left(l_{q, q^{\prime}}\right)$ or in $h_{f_{4}}\left(l_{q, q^{\prime}}\right)$. Since both $f_{1}=H^{\prime} \backslash f_{3}$ and $f_{2}=H^{\prime} \backslash f_{4}$ are contained in $V(p) \cap V(r)$, Observation 12 implies in either case that $S$ contains two empty convex quadrilaterals.

If $f_{2} \notin V(r)$ then $V(r)=\left\{f_{4}, f_{1}\right\}$ with $f_{4} \cap f_{1}=r^{\prime}$ (see Fig 11, left). If $x \in h_{f_{4}}\left(l_{q, q^{\prime}}\right) \cap h_{f_{4}}\left(l_{r, r^{\prime}}\right)$ then Observation 9(a) implies that $S$ contains an empty convex pentagon. Let us assume that $x \notin h_{f_{4}}\left(l_{q, q^{\prime}}\right)$, that is, $x \in h_{f_{3}}\left(l_{q, q^{\prime}}\right)$. Since $f_{1}=H^{\prime} \backslash f_{3}$ is contained in $V(p) \cap V(r)$, Observation 12 implies that $S$ contains two empty convex quadrilaterals. Symmetrically, if $x \notin h_{f_{4}}\left(l_{r, r^{\prime}}\right)$, then necessarily $x \in h_{f_{1}}\left(l_{r, r^{\prime}}\right)$. Since $f_{3}=H^{\prime} \backslash f_{1}$ is contained in $V(p) \cap V(q)$, Observation 12 again implies that $S$ contains two empty convex quadrilaterals.

Case $|H|=3$ and $\left|H^{\prime}\right|=3$. Since $\left|H^{\prime}\right|=3$, there are two inner points $x$ and $y$ that do not belong to $H^{\prime}$, that is, $I \backslash H^{\prime}=\{x, y\}$. These two inner points $x$ and $y$ span a line $l_{x, y}$. We say that $l_{x, y}$ intersects a face $f$, if the two vertices of $f$ are on different sides of $l_{x, y}$. Since we assume all points to be in general position, $l_{x, y}$ intersects exactly two of the three faces of $P$. Before we begin with a detailed case analysis we collect some additional observations. 

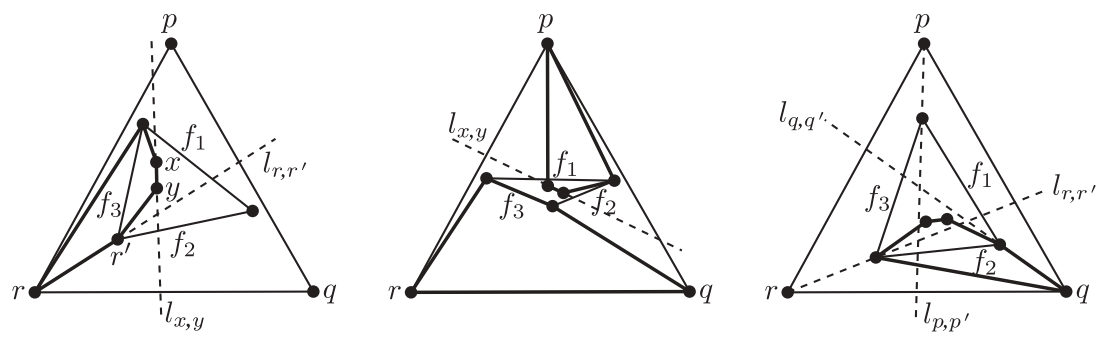

Fig. 12. $|H|=3$ and $\left|H^{\prime}\right|=3$

Observation 13. (a) If $V(p)=\{f\}$ and $l_{x, y}$ does not intersect $f$, then $p, x, y$ and the two vertices of $f$ form an empty convex pentagon.

(b) If $V(p)=\left\{f, f^{\prime}\right\}, x, y \in h_{f}\left(l_{p, p^{\prime}}\right)$ and $l_{x, y}$ does not intersect $f$, then $p, x, y$ and the two vertices of $f$ form an empty convex pentagon.

Lemma 4 implies that $|V(p)| \leq 2$ for every $p \in H$. We distinguish the subcases by the number of outer vertices that see two faces of $\mathrm{P}$.

$|V(p)|=1$ for every $p \in H$.

Every face $f$ of $P$ is seen by exactly one outer vertex. Since $l_{x, y}$ does intersect only two of the three faces of $P$, Observation 13(a) implies that $S$ contains an empty convex pentagon.

$|V(p)|=|V(q)|=1$ for exactly two outer vertices $p$ and $q$, see Fig. 12(left).

If $V(p)=V(q)$, then Observation 7 implies that $S$ contains an empty convex pentagon. We can therefore assume that $V(p) \neq V(q)$. Let $r$ denote the remaining outer vertex, let $V(p)=\left\{f_{1}\right\}$, and let $V(q)=\left\{f_{2}\right\}$. W.1.o.g. we assume that $V(r)=\left\{f_{2}, f_{3}\right\}$ with $r^{\prime}=f_{2} \cap f_{3}$.

If $l_{x, y}$ does not intersect either $f_{1}$ or $f_{2}$ then Observation 13(a) implies that $S$ contains an empty convex pentagon. Hence we can assume that $l_{x, y}$ does intersect both $f_{1}$ and $f_{2}$ and therefore does not intersect $f_{3}$. If either $x \in h_{f_{2}}\left(l_{r, r^{\prime}}\right)$ or $y \in h_{f_{2}}\left(l_{r, r^{\prime}}\right)$ then Observation 9(b) implies that $S$ contains an empty convex pentagon. So we can assume that $x, y \in h_{f_{3}}\left(l_{r, r^{\prime}}\right)$ and then Observation 13(b) implies that $S$ contains an empty convex pentagon.

$|V(p)|=1$ for exactly one outer vertex $p$, Fig. 12 (middle).

Let $V(p)=\left\{f_{1}\right\}$ and denote by $q$ and $r$ the remaining outer vertices. If $l_{x, y}$ does not intersect $f_{1}$ then Observation 13(a) implies that $S$ contains an empty convex pentagon. Hence we can assume that $l_{x, y}$ does intersect $f_{1}$.

If $V(q)=V(r)$ then necessarily $V(q)=V(r)=\left\{f_{2}, f_{3}\right\}$. We can assume w.1.o.g. that $l_{x, y}$ intersects $f_{2}$. Let $p^{\prime}=f_{1} \cap f_{2} . x, y, p$ and $p^{\prime}$ form an convex quadrilateral. Since $H^{\prime} \backslash p^{\prime}=f_{3} \in V(q) \cap V(r)$, Observation 1 implies that the remaining four vertices from a convex quadrilateral as well.

If $V(q) \neq V(r)$ then we can assume that $V(q)=\left\{f_{1}, f_{2}\right\}$ with $q^{\prime}=f_{1} \cap f_{2}$ and $V(r)=\left\{f_{2}, f_{3}\right\}$ with $r^{\prime}=f_{2} \cap f_{3}$. If either $x$ or $y$ are contained in $h_{f_{1}}\left(l_{q, q^{\prime}}\right)$ then Observation 9(b) implies that $S$ contains an empty convex pentagon. So we can assume that both $x$ and $y$ are contained in $h_{f_{2}}\left(l_{q, q^{\prime}}\right)$. Now if either $x$ or $y$ are 

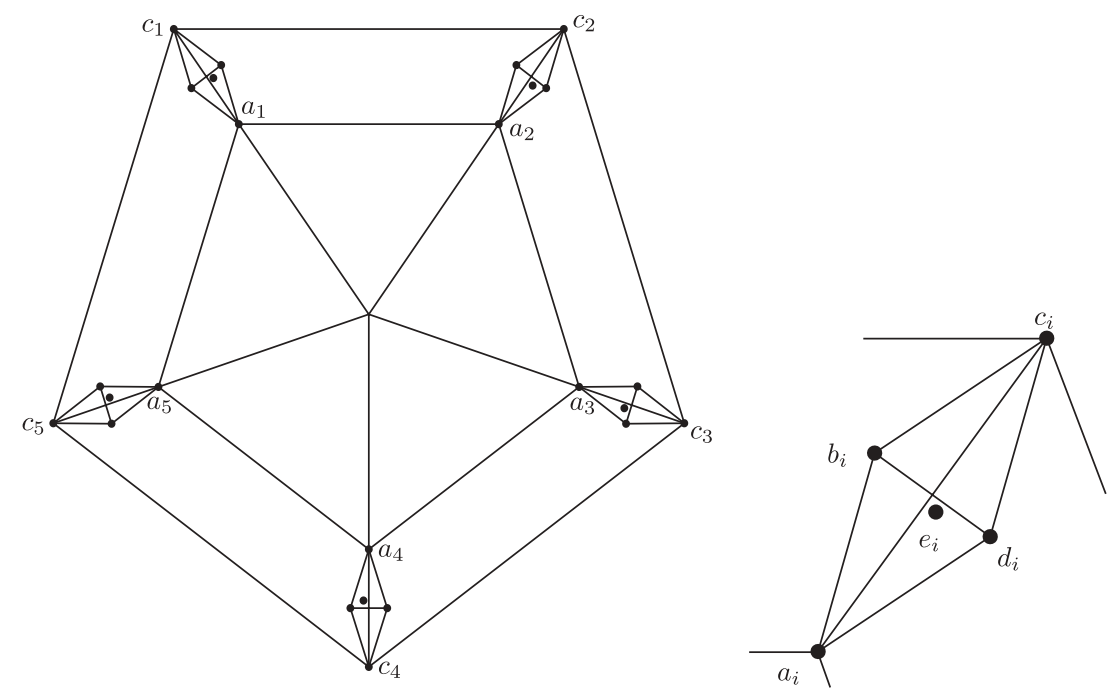

Fig. 13. Our construction for $k=5$ with 25 points on the left. The sub-configuration of $\left\{a_{i}, b_{i}, c_{i}, d_{i}, e_{i}\right\}$ on the right

contained in $h_{f_{2}}\left(l_{r, r^{\prime}}\right)$ then Observation 9(a) implies that $S$ contains an empty convex pentagon. Thus we have $x, y \in h_{f_{2}}\left(l_{q, q^{\prime}}\right) \cap h_{f_{3}}\left(l_{r, r^{\prime}}\right)$. Since $l_{x, y}$ intersects $f_{1}$, it can intersect only one of $f_{2}$ and $f_{3}$ and hence Observation 13(b) implies that $S$ contains an empty convex pentagon.

$|V(p)|=2$ for every $p \in H$, Fig. 12 (right).

Let us denote the three outer vertices with $p, q$, and $r$. W.1.o.g. let $V(p)=\left\{f_{3}, f_{1}\right\}$ with $p^{\prime}=f_{3} \cap f_{1}, V(q)=\left\{f_{1}, f_{2}\right\}$ with $q^{\prime}=f_{1} \cap f_{2}$, and $V(r)=\left\{f_{2}, f_{3}\right\}$ with $r^{\prime}=f_{2} \cap f_{3}$. If either $x$ or $y$ is contained in either of $h_{f_{1}}\left(l_{p, p^{\prime}}\right) \cap h_{f_{1}}\left(l_{q, q^{\prime}}\right)$, $h_{f_{2}}\left(l_{q, q^{\prime}}\right) \cap h_{f_{2}}\left(l_{r, r^{\prime}}\right)$, or $h_{f_{3}}\left(l_{r, r^{\prime}}\right) \cap h_{f_{3}}\left(l_{p, p^{\prime}}\right)$ then Observation 9(a) implies that $S$ contains an empty convex pentagon. We therefore assume that $x, y \in h_{f_{1}}\left(l_{p, p^{\prime}}\right) \cap$ $h_{f_{2}}\left(l_{q, q^{\prime}}\right) \cap h_{f_{3}}\left(l_{r, r^{\prime}}\right)$ or $x, y \in h_{f_{3}}\left(l_{p, p^{\prime}}\right) \cap h_{f_{1}}\left(l_{q, q^{\prime}}\right) \cap h_{f_{2}}\left(l_{r, r^{\prime}}\right)$. In either case, since $l_{x, y}$ intersects only two out of $f_{1}, f_{2}$, and $f_{3}$, Observation 13(b) implies that $S$ contains an empty convex pentagon.

\subsection{Lower Bound}

We present a lower bound construction of $5 k$ points for every odd $k \geq 3$ such that any pseudo-convex decomposition consists of at least $3 k-1$ faces (see Fig. 13). This implies

$$
\psi_{d}(n) \geq 3\left\lfloor\frac{n}{5}\right\rfloor-1
$$

Lemma 5. For every odd $k$, there are $5 k$ points in the plane such that every pseudoconvex decomposition consists of at least $3 k-1$ faces. 
Description of Our Construction. For every odd $k \in \mathbb{N}$, we construct a set of $5 k$ points $S_{k}=\left\{a_{i}, b_{i}, c_{i}, d_{i}, e_{i},: i=1,2, \ldots, k\right\}$. The polygons $A=a_{1} a_{2} \ldots a_{k}$ and $C=c_{1} c_{2} \ldots c_{k}$ form two centrally symmetric regular $k$-gons such that $A \subset C$. Let $o$ denote their center of symmetry. For every $i=1,2 \ldots, k$, the quadrilateral $Q_{i}=a_{i} b_{i} c_{i} d_{i}$ is a rhombus, where the diagonal $a_{i} c_{i}$ is much longer than $b_{i} d_{i}$. Point $e_{i}$ lies near the center of the rhombus $a_{i} b_{i} c_{i} d_{i}$ in the interior of the triangle $a_{i} b_{i} d_{i} \cap a_{i} c_{i} d_{i}$. The configurations $\left\{a_{i}, b_{i}, c_{i}, d_{i}, e_{i}\right\}, i=1,2, \ldots, k$, are congruent. See Fig. 13 for an example with $k=5$. The ratio of the diameter of the polygons $A$ and $C$ are so close to 1 that any rhombus $Q_{i}$ can be separated from the other rhombi by a straight line. Furthermore, we choose the ratio of the two diagonals of $Q_{i}$ such that every line passing through $a_{i}$ (resp., $c_{i}$ ) and another point of $\left\{a_{i}, b_{i}, c_{i}, d_{i}, e_{i}\right\}$ passes through between the rhombi $Q_{j}$ and $Q_{j+1}$ for $j=i+\frac{k-1}{2} \bmod k$. The point $e_{i}$ is so close to the midpoint of $b_{i} d_{i}$ that (1) every line spanned by any two points in $\left\{b_{i}, d_{i}, e_{i}\right\}$ intersects the segments $c_{i-1} c_{i}$ and $c_{i} c_{i+1}$; (2) for every vertex $x \in S_{k} \backslash\left\{b_{i}, d_{i}, e_{i}\right\}$, the half-line $x e_{i}$ lies in the angular domain $\angle b_{i} x d_{i}<\pi$.

Reference Points. For a point set $S_{k}$ and a pseudo-convex decomposition $D$, we choose $6 k$ reference points and show that every face of $D$, with at most one exception, contains at most two reference points; and the exceptional face may contain up to four reference points. This proves that $|D| \geq 3 k-1$.

Let $\varepsilon>0$ be a sufficiently small real number. Each reference point lies in the $\varepsilon$-neighborhood of the intersection point of two lines spanned by $S_{k}$, in the interior of a triangle incident to the intersection point. The locations of six different types of reference points are given in Table 2 below.

The location of the reference points, however, slightly depends on the decomposition $D$. The reference point $x_{i}$ lies in the triangle $\Delta\left(b_{i}, c_{i}, b_{i} d_{i} \cap a_{i} c_{i}\right)$ if the pseudotriangle $b_{i} d_{i} c_{i} a_{i+1} e_{i}$ or $b_{i} c_{i} a_{i-1} e_{i} d_{i}$ is in $D$; otherwise $x_{i}$ lies in $\Delta\left(a_{i}, b_{i}, b_{i} d_{i} \cap\right.$ $\left.a_{i} c_{i}\right)$. Also, if a pseudo-triangle in $D$ has consecutive vertices at $\left(a_{i}, d_{i}, c_{i}, c_{i+1}\right)$ or $\left(a_{i}, e_{i}, c_{i}, d_{i}, c_{i+1}\right)$, it has at least one additional vertex, it but does not contain the center $o$, then move the reference point $v_{i}$ to $w_{i}$ (it can be thought of as a double reference point at $w_{i}$ ). Similarly, if a pseudo-triangle in $D$ has consecutive vertices at $\left(c_{i}, c_{i+1}, b_{i+1}, a_{i+1}\right)$, has at least five vertices, but does not contain $o$, then we move $w_{i}$ to $v_{i}$.

Proof. We show that all but at most one faces contain at most two reference points. One can show that a face in $D$ contains three or four reference points only if it also contains the symmetry center $o$ of the construction (Fig. 15(f)). No face in

Table 2. The locations of the six types of reference points for $i=1,2, \ldots, k$ (addition is $\bmod k$ )

\begin{tabular}{lll}
\hline Reference point & in the $\varepsilon$-neighborhood of & in the triangle \\
\hline$x_{i}$ & $b_{i} d_{i} \cap a_{i} c_{i}$ & $\Delta\left(a_{i}, b_{i}, b_{i} d_{i} \cap a_{i} c_{i}\right)$ or $\Delta\left(b_{i}, c_{i}, b_{i} d_{i} \cap a_{i} c_{i}\right)$ \\
$y_{i}$ & $b_{i} d_{i} \cap c_{i} e_{i}$ & $\Delta\left(d_{i}, e_{i}, b_{i} d_{i} \cap c_{i} e_{i}\right)$ \\
$z_{i}$ & $c_{i} b_{i+1} \cap d_{i} c_{i+1}$ & $\Delta\left(c_{i}, c_{i+1}, c_{i} b_{i+1} \cap d_{i} c_{i+1}\right)$ \\
$u_{i}$ & $c_{i} d_{i+1} \cap b_{i} c_{i+1}$ & $\Delta\left(b_{i}, d_{i+1}, c_{i} d_{i+1} \cap b_{i} c_{i+1}\right)$ \\
$v_{i}$ & $a_{i} c_{i+1} \cap b_{i} a_{i+(k-1) / 2}$ & $\Delta\left(a_{i}, a_{i+(k-1) / 2}, a_{i} c_{i+1} \cap b_{i} a_{i+(k-1) / 2}\right)$ \\
$w_{i}$ & $c_{i} a_{i+1} \cap d_{i+1} a_{i+1+(k+1) / 2}$ & $\Delta\left(a_{i+1}, a_{i+1+(k+1) / 2}, c_{i} a_{i+1} \cap d_{i+1} a_{i+1+(k+1) / 2}\right)$ \\
\hline
\end{tabular}




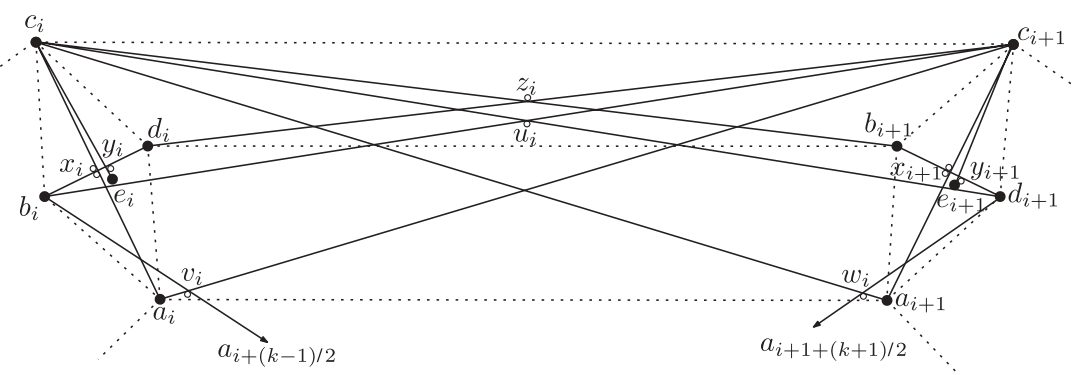

Fig. 14. The location of the reference points for sub-configurations $Q_{i}$ and $Q_{i+1}$
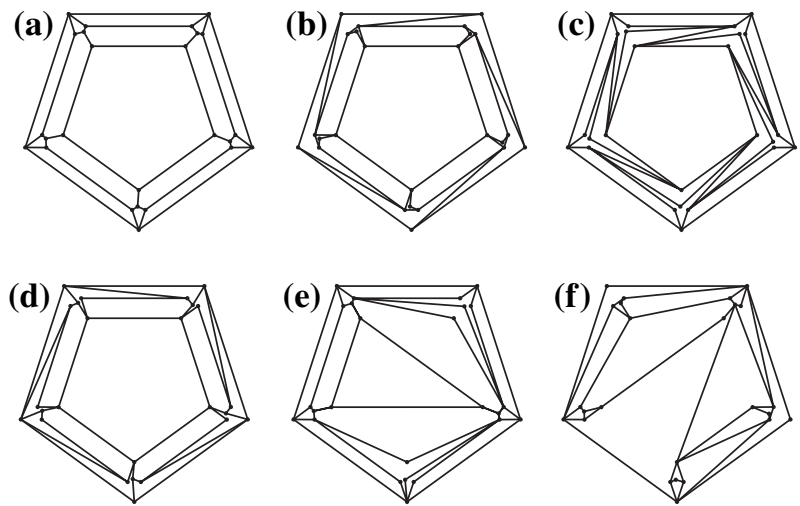

Fig. 15. Five tilings of the convex hull of $S_{5}$ with 16 convex or pseudo-triangle faces (a-e), and one with 17 faces (f)

$D$ contains more than four reference points. It is not too difficult to verify these statements by classifying all possible faces of a pseudo-convex decomposition $D$.

It is easy to find all possible maximal convex faces spanned by $S_{k}$. We have the regular $k$-gon $a_{1} a_{2} \ldots a_{k}$. For every $i=1,2, \ldots, k$, we have $a_{i} b_{i} c_{i} e_{i}, b_{i} c_{i} d_{i} e_{i}$ determined by a single group. In two consecutive groups, we have all nine combinations of two maximal convex arcs from the two groups: one of the three $\operatorname{arcs} a_{i} e_{i} d_{i}, a_{i} b_{i} e_{i}$, and $d_{i} c_{i}$ from group $i$ with one of the three arcs $b_{i+1} e_{i+1} a_{i+1}, e_{i+1} d_{i+1} a_{i+1}$, and $c_{i+1} b_{i+1}$ from group $i+1$, for $i=1,2, \ldots, k$. Two such maximal convex arcs from two nonconsecutive groups $i$ and $j$, with $1<j-i<k / 2 \bmod k$, also form a maximal convex polygon, which passes through the intermediate $a$ vertices (for instance, the combination of the first convex arcs listed above gives $a_{i} e_{i} d_{i} a_{i+1} \ldots a_{j-1} b_{j} e_{j} a_{j}$ ). Every convex face contains at most two reference points.

One can also classify all empty pseudo-triangles spanned by $S_{k}$. Every pseudotriangle is determined by three corners and their bisectors. In our construction, all vertices of a pseudo-triangle must lie in at most three groups, those containing the three corners. A pseudo-triangle can contain the reference points $z_{i}, u_{i}, v_{i}$, or $w_{i}$, only if it has a corner in both groups $i$ and $i+1$. A pseudo-triangle can contain $x_{i}$ or $y_{i}$ if it has either two corners in group $i$, or one corner in group $i$ and contains 
the center $o$. See a few examples in Fig. 15. A systematic search of possible pseudotriangle faces confirms that every pseudo-triangle contains at most two reference points unless it contains the center of symmetry $o$.

\section{Pseudo-Convex Partitions}

An upper bound of $\psi_{p}(n) \leq\lceil n / 4\rceil$ can be easily established: Partition the point set by vertical lines into groups of size four, allowing at most one group to be smaller if $n$ is not a multiple of four - every four points forms either a convex quadrilateral or a pseudo-triangle. Any better bound than $\lceil n / 4\rceil$ for some $n \in \mathbb{N}$ may improve this upper bound. For example, we do not know the exact value of $\psi_{p}(13)$, we only know that $\psi_{p}(13) \in\{3,4\}$ (c.f., Table 1$) . \psi_{p}(13)=3$ would imply $\psi_{p}(n) \leq\lceil 3 n / 13\rceil$ by partitioning the point set into groups of size 13 and partitioning the groups independently.

\subsection{Lower Bound}

Lemma 6. $\psi_{p}(n) \geq \frac{3}{4}\left\lfloor\frac{n}{4}\right\rfloor$.

Proof. We consider a set $S$ of $n=4 k$ points illustrated in Fig. 16. $S$ consists of $k$ groups of 4 points, $a_{i}, b_{i}, c_{i}$, and $d_{i}$. First we show that if $c_{i}$ is a reflex vertex of a pseudo-triangle $P$, then $a_{i}$ and $b_{i}$ must be the corners of $P$ : This is the case since $c_{i}$ lies in the convex hull of the corners of $P$, and there is a halfplane for $a_{i}\left(b_{i}\right)$ whose boundary line passes through $c_{i}$ and whose intersection with $P$ is $a_{i}\left(b_{i}\right)$.

Let $W \subset S$ denote a subset of $3 k$ points $\left\{a_{i}, b_{i}, c_{i}: i=1,2, \ldots, k\right\}$. Consider a polygon $P$ from a pseudo-convex partition of $S$. We show next that $P$ is incident to at most 4 points of $W$. This implies immediately that every pseudo-convex partition of $S$ consists of at least $3 k / 4=3 n / 16$ polygons. Suppose, by contradiction, that $P$ is incident to more than 4 points of $W$.

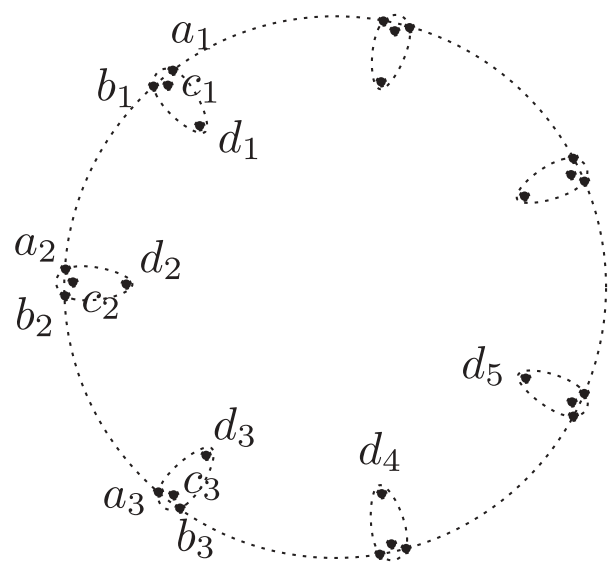

Fig. 16. Our lower bound construction for $k=7$ 
First suppose that $P$ is convex, that is, $P$ contains a convex pentagon $Q$ with all vertices in $W$. Since each group contains only three points of $W, Q$ must have corners in at least two groups. $Q$ can contain at most two points from each group, because the triangle $a_{i} b_{i} c_{i}$ cannot be completed to a convex pentagon in $S$. Therefore, $Q$ must have corners in at least three groups, and it contains a triangle $T$ with corners of $W$ from three different groups. There is a group $i \in\{1,2, \ldots, k\}$ such that $T$ has a corner in group $i$ and the other two corners of $T$ are in a group $j \in[i+1, i+\lfloor k / 2\rfloor$ $\bmod k]$ and in a group $j^{\prime} \in[i+\lceil k / 2\rceil \bmod k, i+k-1 \bmod k]$. It follows that $T$ contains $d_{i}$ in its interior, a contradiction.

If $P$ is a pseudo-triangle with at least five vertices in $W$, then it must have at least two reflex vertices in $W$. Since the convex hull vertices can only be corners of $P$, at least two reflex vertices are some $c_{i}$ and $c_{j}, i \neq j$. We have seen that if $P$ contains $c_{i}$ and $c_{j}$, then it also contains $a_{i}, b_{i}$ and $a_{j}, b_{j}$, and so it must have four corners: A contradiction.

\section{Pseudo-Convex Coverings for Small Point Sets}

We start with a simple observation about three points lying in the interior of a triangle. Then we show that every set of 12 points contains a hexagon that is either convex or a pseudo-triangle.

Lemma 7. Every set $S$ of 6 points whose convex hull is a triangle has a spanning pseudo-triangle.

Proof. Let $c_{1}, c_{2}$, and $c_{3}$, be three vertices of of the convex hull of $S$ sorted in clockwise order; and let $p_{1}, p_{2}$, and $p_{3}$ be the inner vertices of $S$, sorted clockwise around $c_{1}$. Then we first form two side chains $c_{1}, p_{1}, c_{2}$ and $c_{1}, p_{3}, c_{3}$, respectively, which do not interfere. If $p_{2}$ lies to the left of the directed line $c_{2}, p_{1}$ (resp., right of the directed line $c_{3}, p_{3}$ ), then we extend the side chain $c_{1}, p_{1}, c_{2}$ to $c_{1}, p_{2}, p_{1}, c_{2}$ (resp., $c_{1}, p_{3}, c_{3}$ to $c_{1}, p_{2}, p_{3}, c_{3}$ ). In the remaining case $p_{2}$ lies to the right of the directed line $c_{3}, p_{3}$ and to the left of the directed line $c_{2}, p_{1}$ and we therefore take the side chain $c_{2}, p_{2}, c_{3}$. In all cases we get a valid pseudo-triangle which spans all 6 vertices of $S$.

Lemma 8. Every set $S$ of $n$ points contains either a convex hexagon or a pseudo-triangle with 6 vertices if and only if $n \geq 12$.

Proof. Let $h$ be the size of the convex hull of $S$. We prove the statement by a case analysis over various values of $h$. If $h=3$, then the theorem directly follows from Lemma 7. Note that additional inner points are allowed in Lemma 7, as we do not require the hexagon or pseudo-triangle to be empty. For $h=4,5$, decompose the convex hull of $S$ into two (three, respectively) triangles. At least one triangle must contain 3 or more interior points. Again the statement follows from Lemma 7 applied to this triangle. If $h \geq 6$, then $S$ certainly contains a convex hexagon. 


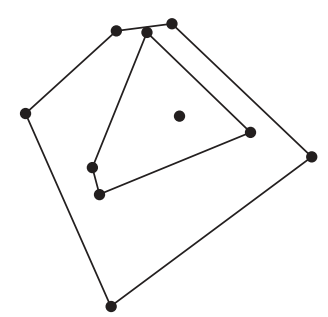

Fig. 17. A configuration of 10 points whose pseudo-convex cover consists of at least 3 polygons

There exist precisely 9 (out of over 2.33 billion) realizable order types of 11 points which do not contain a convex hexagon nor a pseudo-triangle with 6 vertices ${ }^{1}$. Thus the bound is tight with respect to $n$.

Lemma 9. $\psi_{c}(n)=3$ for $n=10, \ldots, 15$.

Proof. For $n=10, \ldots, 14$, we obtain $\psi_{c}(n) \leq 3$ from the fact that every set of $n \geq 9$ points contains a convex pentagon together with $\psi_{c}(5)=2, \ldots, \psi_{c}(9)=2$. The matching lower bound $\psi_{c}(10) \geq 3$ (and thus $\psi_{c}(11) \geq 3, \ldots, \psi_{c}(14) \geq 3$ ) follows from a configuration of 10 points whose pseudo-convex cover consists of at least 3 polygons. We found this configuration with the help of the order type data base and to our surprise there is only one set (out of 14, 309, 547 order types) which has this property. Here are its coordinates: $(0,43470),(20468,62019),(27350,61551)$, (32984,63477), (34692,42743), (50624,39069), (64372,33534), (15064,31131), (16660,25083), (19152,0) (see Fig. 17). We obtain $\psi_{c}(15)=3$ from Lemma 8 together with $\psi_{c}(9)=2$.

\section{Pseudo-Convex Decompositions of the Interior of a Simple Polygon}

Theorem 4. Every simple polygon with $n \geq 3$ vertices has a decomposition into at most $\left\lceil\frac{n-2}{2}\right\rceil$ convex or pseudo-triangular faces, and this bound is the best possible.

Proof. The lower bound is attained by the comb polygons (Fig. 18 (a)). We prove the upper bound by induction on $n \in \mathbb{N}$. The theorem is obvious for $n=3,4$. Consider a simple polygon $P_{n}$ with $n \geq 5$ vertices. Triangulate $P_{n}$ and let $T_{n}$ denote the dual graph of the triangulation. Every node of $T_{n}$ corresponds to a triangle, and every edge of $T_{n}$ corresponds to a diagonal in the triangulation. $T_{n}$ is a tree with maximal degree three and with $n-2$ nodes.

If $n$ is odd then we delete a triangle $t$ corresponding to a leaf node in $T_{n}$. By induction, $P_{n}-t$ can be decomposed into $\frac{n-3}{2}$ faces. Therefore $P_{n}$ decomposes into $\frac{n-3}{2}+1=\left\lceil\frac{n-2}{2}\right\rceil$ faces.

1 Let us note here that the probability of winning the Jackpot of the Austrian lottery (6 out of 45 ) is about 30 times higher than the probability of finding such a set at random. 
(a)

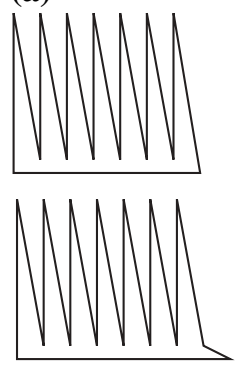

The comb polygon for $n$ odd and for $n$ even. (b)

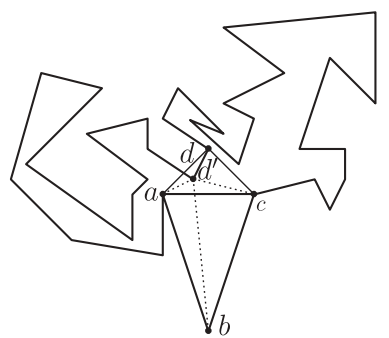

If $b$ is incident to a diagonal $b d$, then there is a vertex $d^{\prime}$ such that $b d^{\prime}$ and at least one of $a d^{\prime}$ or $c d^{\prime}$ are also diagonals. (c)

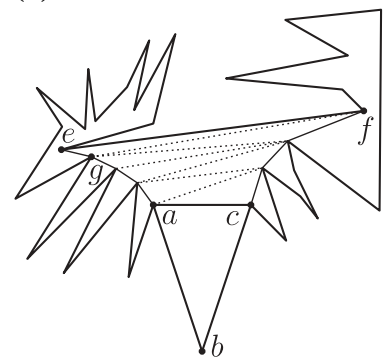

If $b$ sees the edge $e f$ then we can form the pseudotriangle $\operatorname{pt}(b, e, f)$.

Fig. 18. Lower bound (a). An example 24-g on. (b)-(c)

Assume that $n$ is even, and so $\left[\frac{n-2}{2}\right]=\frac{n}{2}-1$. The triangulation consists of an even number of triangles. If a diagonal decomposes $P_{n}$ into two even polygons, then induction completes the proof. Hence we assume that every diagonal decomposes $P_{n}$ into two odd polygons.

Let the triangle $a b c$ correspond to a leaf in $T_{n}$ such that $a c$ is a diagonal of $P_{n}$. We show that no diagonal of $P_{n}$ is incident to $b$. Suppose, by contradiction, that $b d$ is a diagonal of $P_{n}$ (Fig. 18 (b)). Then abcd must be a convex polygon lying in $P_{n}$. Let $d^{\prime}$ be the vertex of $P_{n}$ in $a c d \backslash\{a, c\}$ closest to the line $a c$. Note that $b d^{\prime}$ is a diagonal of $P_{n}$, and at least one of $a d^{\prime}$ and $c d^{\prime}$ is also a diagonal (since $n \geq 5$ ). If $b d^{\prime}$ decomposes $P_{n}$ into odd polygons, then either $a d^{\prime}$ or $c d^{\prime}$ decomposes it into two (non-empty) even polygons. We conclude that $b$ is not incident to any diagonal of $P_{n}$ and so it sees the interior of a unique edge $e f$ of $P_{n}$ (Fig. 18 (c)).

Consider the pseudo-triangle $\operatorname{pt}(b, e, f)$, which is the unique pseudo-triangle lying in $P_{n}$ with corners $b, e$, and $f$. If $P_{n}=\operatorname{pt}(b, e, f)$, then $P_{n}$ is a pseudo-triangle, and our proof is complete. Each of the components of $P_{n}-\operatorname{pt}(b, e, f)$ is an odd polygon. Every such component is adjacent to a unique edge of the geodesic geo $(a, e)$ or geo $(c, f)$. If $\operatorname{pt}(b, e, f)$ has $k$ vertices, then it has $k-3$ edges along these geodesics (all edges except $a b, b c$, and $e f$ ). We show that there is one edge along the geodesics geo $(a, e)$ and $\operatorname{geo}(c, f)$ that is not adjacent to any component of $P_{n}-\operatorname{pt}(b, e, f)$ : Consider the dual graph of an arbitrary triangulation of $\mathrm{pt}(b, e, f)$. It is a tree where one leaf node corresponds to $a b c$ and another leaf corresponds to ef $g$ for some vertex $g$. Assume w.l.o.g. that $e g$ is a side and $f g$ is a diagonal in $\operatorname{pt}(b, e, f)$. If $e g$ were adjacent to an odd component of $P_{n}-\mathrm{pt}(b, e, f)$, then $f g$ would partition $P_{n}$ into two even polygons. Therefore $\operatorname{pt}(b, e, f)$ with $k$ vertices is adjacent to at most $k-4$ components of $P_{n}-\operatorname{pt}(b, e, f)$.

Let $n_{i}$ denote the number of vertices of the components of $P_{n}-\operatorname{pt}(b, e, f)$ for $i=1,2, \ldots, k-4$. We have $k+\sum_{i=1}^{k-4}\left(n_{i}-2\right)=n$. By induction, every odd component with $n_{i}$ vertices can be decomposed into $\left(n_{i}-1\right) / 2$ faces. Together with 
$\operatorname{pt}(b, e, f)$, the polygon $P_{n}$ can be decomposed into

$$
1+\sum_{i=1}^{k-4} \frac{n_{i}-1}{2} \leq 1+\frac{1}{2}\left(\sum_{i=1}^{k-4} n_{i}-2\right)+\frac{k-4}{2}=\frac{n}{2}-1
$$

faces, as required.

\section{Conclusions and Open Problems}

We proposed pseudo-convex decompositions, partitions, and coverings. We established some of their basic properties and gave combinatorial bounds on their complexity. Our upper bounds depend on new Ramsey-type results concerning disjoint empty convex $k$-gons in the plane. We (obviously) would like to know what the exact bounds on $\psi_{d}(n)$ and $\psi_{p}(n)$ are and if the exact bound for $\psi_{d}(n)$ can be realized with a pointed decomposition. It would also be interesting to determine the complexity of computing a minimum pseudo-convex decomposition or covering for a given point set.

Acknowledgements. The first two authors thank Ferran Hurtado and Hannes Krasser for valuable discussions over the matter presented here.

\section{References}

1. Agarwal, P.K., Basch, J., Guibas, L.J., Hershberger, J., Zhang, L.: Deformable free space tilings for kinetic collision detection. Int. J. Robot. Res. 21, 179-197 (2002)

2. Aichholzer, O., Aurenhammer, F., Krasser, H.: Enumerating order types for small point sets with applications. Order 19, 265-281 (2002)

3. Aichholzer, O., Krasser, H.: Abstract order type extensions and new results on the rectilinear crossing number, in: Proc. 21st Sympos. Comput. Geom. pp. 91-98, ACM Press (2005)

4. Arkin, E.M., Fekete, S.P., Hurtado, F., Mitchell, J.S.B., Noy, M., Sacristán, V., Sethia, S.: On the reflexivity of point sets, in: Discrete and Computational Geometry: The Goodman-Pollack Festschrift (B. Aronov et al., eds.), vol. 25 of Algorithms Combin., pp. 139-156, Springer, Berlin (2003)

5. Chazelle, B., Dobkin, D.: Optimal convex decompositions, in: Computational Geometry, (G.T. Toussaint, ed.), pp. 63-133, North-Holland, Amsterdam (1985)

6. Chazelle, B., Edelsbrunner, H., Grigni, M., Guibas, L.J., Hershberger, J., Sharir, M., Snoeyink, J.: Ray shooting in polygons using geodesic triangulations. Algorithmica 12, 54-68 (1994)

7. de Berg, M., Matoušek, J., Schwarzkopf, O.: Piecewise linear paths among convex obstacles. Discrete Comput. Geom. 14(1), 9-29 (1995)

8. Erdős, P., Szekeres, G.: A combinatorial problem in geometry. Comp. Math. 2, 463-470 (1935)

9. Erdős, P., Szekeres, G.: On some extremum problem in geometry. Ann. Univ. Sci. Budapest, 3-4:53-62 (1960)

10. Fevens, T., Meijer, H., Rappaport, D.: Minimum convex partition of a constrained point set. Discrete Appl. Math. 109, 95-107 (2001)

11. García-López, J., Nicolás, M.: Planar point sets with large minimum convex partitions, in: Abstracts 22nd European Workshop on Comput. Geom., pp. 51-54, Delphi (2006) 
12. Gerdjikov, S., Wolff, A.: Pseudo-convex decomposition of simple polygons, in: Abstracts 22nd European Workshop on Computational Geometry, pp. 13-16, Delphi (2006)

13. Gerken, T.: On empty convex hexagons in planar point sets, 2005, submitted

14. Goodman, J., Pollack, R.: Allowable sequences and order types in discrete and computational geometry, in: New Trends in Discrete and Computational Geometry, pp. 103-134, Springer, New York (1993)

15. Goodrich, M., Tamassia, R.: Dynamic ray shooting and shortest paths in planar subdivision via balanced geodesic triangulations. J. Algorithms 23, 51-73 (1997)

16. Haas, R., Orden, D., Rote, G., Santos, F., Servatius, B., Servatius, H., Souvaine, D., Streinu, I., Whiteley, W.: Planar minimally rigid graphs and pseudo-triangulations, Comput. Geom. Theory Appl. 31(1-2), 31-61 (2005)

17. Harborth, H.: Konvexe Fünfecke in ebenen Punktmengen, Elemente Math. 33(5), 116118 (1978)

18. Hosono, K., Urabe, M.: On the number of disjoint convex quadrilaterals for a planar point set. Comput. Geom. Theory Appl. 20, 97-104 (2001)

19. Károlyi, Gy., Pach, J., Tóth, G.: Ramsey-type results for geometric graphs I. Discrete Comput. Geom. 18, 247-255 (1997)

20. Károlyi, Gy., Pach, J., Tóth, G., Valtr, P.: Ramsey-type results for geometric graphs II. Discrete Comput. Geom. 20, 375-388 (1998)

21. Keil, J.M.: Decomposing a polygon into simpler components, SIAM J. Comput. 14, 799-817 (1985)

22. Keil, J.M., Snoeyink, J.: On the time bound for convex decomposition of simple polygons. Int. J. Comput. Geom. Appl. 12, 181-192 (2002)

23. Kirkpatrick, D., Speckmann, B.: Kinetic maintenance of context-sensitive hierarchical representations for disjoint simple polygons, In: Proc. 18th Sympos. Comput. Geom., pp. 179-188, ACM Press (2002)

24. Larman, D., Matoušek, J., Pach, J., Töröcsik, J.: A Ramsey-type result for planar convex sets, Bull. Lond. Math. Soc. 26, 132-136 (1994)

25. Lien, J.-M., Amato, N.M.: Approximate convex decomposition, In: Proc. 20th Sympos. Comput. Geom., pp. 17-26, ACM Press (2004)

26. Neumann-Lara, V., Rivera-Campo, E., Urrutia, J.: A note on convex decompositions of a set of points in the plane, Graphs Combin. 20(2), 223-231 (2004)

27. O'Rourke, J.: Visibility, in: Handbook of Discrete and Computational Geometry, (2nd edn.), pp. 643-664, CRC Press, Boca Raton, FL (1997)

28. Pocchiola, M., Vegter, G.: Topologically sweeping visibility complexes via pseudotriangulations. Discrete Comput. Geom. 16, 419-453 (1996)

29. Pocchiola, M., Vegter, G.: Minimal tangent visibility graphs. Comput. Geom. Theory Appl. 6, 303-314 (1996)

30. Ramaswami, S., Ramos, P.A., Toussaint, G.T.: Converting triangulations to quadrangulations. Comput. Geom. Theory Appl. 9, 257-276 (1998)

31. Spillner, A.: Optimal convex partitions of point sets with few inner points, In: Proc. 17th Canadian Conf. Comput. Geom., pp. 39-42, Windsor, ON (2005)

32. Streinu, I.: A combinatorial approach to planar non-colliding robot arm motion planning, in: Proc. 41st Sympos. Foundations Comp. Sci., pp. 443-453, IEEE Press (2000)

33. Urabe, M.: On a partition into convex polygons. Discrete Appl. Math. 64, 179-191 (1996)

34. Valtr, P.: On empty hexagons, 2006, submitted 